\title{
ON THE FACTORIZATION OF MEROMORPHIC MELLIN SYMBOLS
}

\author{
INGO WITT \\ University of Potsdam
}

February 27, 2001

\begin{abstract}
It is proved that meromorphic, parameter-dependent elliptic Mellin symbols can be factorized in a particular way. The proof depends on the availability of logarithms of pseudodifferential operators. As a byproduct, we obtain a characterization of the group generated by pseudodifferential operators admitting a logarithm. The factorization has applications to the theory of pseudodifferential operators on spaces with conical singularities, e.g., to the index theory and the construction of various sub-calculi of the cone calculus.
\end{abstract}

\section{Contents}

1. Introduction

2. Preliminaries

2.1 Parameter-dependent operators

2.2 Meromorphic Mellin symbols

2.3 Reduction to holomorphic Mellin symbols

3. Logarithms of pseudodifferential operators

3.1 The classes $L^{\mu \log }(X ; \Lambda)$

3.2 The exponential map

3.3 The topological invariant $\Psi(A)$

3.4 Characterization of the image of exp

4. The kernel cut-off technique

5. Proof of the main theorem

5.1 Beginning of the proof

5.2 Continuation of the proof

5.3 The remaining case for $\operatorname{dim} X=1$

1991 Mathematics Subject Classification. Primary 35S05; Secondary 17B65, 47D03.

Key words and phrases. Meromorphic and holomorphic Mellin symbols, logarithms of pseudodifferential operators, image of the exponential map, kernel cut-off technique.

Typeset by $\mathcal{A M}_{\mathcal{M}} \mathrm{T}_{\mathrm{E}} \mathrm{X}$ 


\section{INTRODUCTION}

Meromorphic Mellin symbols arise in questions of characterizing elliptic regularity and the asymptotics of solutions to differential equations on spaces with conical singularities, and other singularities as well, and the construction of pseudodifferential parametrices to elliptic operators. The necessity of studying the functionalanalytic properties of such Mellin symbols has been emphasized by B.-W. Schulze. In that connection, it was asked whether meromorphic Mellin symbols can be factorized in a particular way that is made precise below. In this paper, we give an affirmative answer to that question. This has consequences to the index theory, e.g., [15], and the construction of sub-calculi of Schulze's cone and edge pseudodifferential calculus, respectively, e.g., $[16,17]$, which are then suitable for treating certain non-elliptic problems, e.g., [23].

We formulate the main result of this paper. Since the definition of a meromorphic Mellin symbols requires some technical explanation, we postpone it to Section 2.2. Notice already here that a Mellin symbol $G(z) \in \mathcal{M}_{P}^{\mu}(X)$ is a meromorphic, operator-valued function of $z \in \mathbb{C}$ taking values in the space $L^{\mu}(X)$ of classical pseudodifferential operators of order $\mu$ on the closed $C^{\infty}$-manifold $X$, where the pattern of poles of $G$ including certain information about the coefficients of the principal parts of its Laurent expansions around the poles is encoded in the asymptotic type $P$. In particular, $G(z) \in \mathcal{M}_{\mathcal{O}}^{\mu}(X)$, where $\mathcal{O}$ denotes the empty asymptotic type, means that $G$ is holomorphic on $\mathbb{C}$. Further some parameterdependence along the lines $\Gamma_{\beta}=\{z \in \mathbb{C} ; \Re z=\beta\}, \beta \in \mathbb{R}$, is required.

Theorem 1.1. Let $\mu \in \mathbb{C}, P \in \underline{\operatorname{As}}(X)$ be an asymptotic type, and $G(z) \in \mathcal{M}_{P}^{\mu}(X)$ be an elliptic Mellin symbol. Then there exists a (necessarily elliptic) Mellin symbol $F(z) \in \mathcal{M}_{\mathcal{O}}^{\mu}(X)$ and a Mellin symbol $H(z) \in \mathcal{M}_{Q}^{-\infty}(X)$ for some resulting asymptotic type $Q \in \underline{\operatorname{As}}(X)$ such that $F^{-1}(z) \in \mathcal{M}_{\mathcal{O}}^{-\mu}(X)$ and the multiplicative decomposition

$$
G(z)=F(z)(1+H(z))
$$

holds on the whole of $\mathbb{C}$.

The rest of the paper is devoted to the proof of Theorem 1.1. The proof is constructive. Its main ingredients are logarithms of pseudodifferential operators and the kernel cut-off technique.

Let $L^{\mu}(X ; \Lambda)$ be the class of parameter-dependent classical pseudodifferential operators on a closed $C^{\infty}$-manifold $X$ of order $\mu \in \mathbb{C}$ with parameter space $\Lambda=\mathbb{R}^{l}$, $l \in \mathbb{N}$. (The case $l=1$ is needed for Mellin symbols.) While, in defining logarithms of pseudodifferential operators, one usually introduces powers $A^{\sigma}, \sigma \in \mathbb{C}$, for suitable elliptic operators $A \in L^{\mu}(X ; \Lambda)$ first, cf. [19] and also [5], we immediately introduce the class $L^{\mu \log }(X ; \Lambda)$ of generators $B$ of the (analytic) one-parameter groups $A^{\sigma}, \sigma \in \mathbb{C}$, and afterwards we define the exponential map

$$
\exp : L^{\mu \log }(X ; \Lambda) \rightarrow L^{\mu}(X ; \Lambda) \text {. }
$$


Then this yields, in particular, $A^{\sigma}=\exp (\sigma B), \sigma \in \mathbb{C}$, where $B=\log A$. Here, of course, the problem of determining the image of the map (1.2) (and of inverting the map (1.2) on its image) arises. A sufficient condition for $A \in L^{\mu}(X ; \Lambda)$ to possess a logarithm is Seeley's condition on the spectral cut, cf. [19].

In defining the exponential map (1.2), we make use of a perturbation argument from semigroup theory that amounts in solving an integral equation of Volterra type. In doing so, we exploit the following two facts:

- $\left(1+|\lambda|^{2}-\Delta_{g}\right)^{\mu / 2}$, where $\Delta_{g}$ is the Laplace-Beltrami operator with respect to a Riemannian metric $g$ on $X$, belongs to the class $L^{\mu}(X ; \Lambda)$, cf. $[19,20]$;

- the Fréchet algebra $L^{0}(X ; \Lambda)$ admits a holomorphic functional calculus, cf. $[3,22]$.

In a final step, we characterize the image of the exponential map (1.2) modulo $L^{-\infty}(X ; \Lambda)$. To this end, we introduce a topological invariant $\Psi(A) \in H^{1}\left(\left(T^{*} X \times\right.\right.$ $\Lambda) \backslash 0)$ assigned to each elliptic operator $A \in L^{\mu}(X ; \Lambda)$. Actually, $\Psi(A)$ only depends on the principal symbol $\sigma^{\mu}(A)$ and $\Psi\left(A A^{\prime}\right)=\Psi(A)+\Psi\left(A^{\prime}\right)$ for elliptic $A \in L^{\mu}(X ; \Lambda), A^{\prime} \in L^{\mu^{\prime}}(X ; \Lambda)$. For instance, in case $l=0$, an operator $A \in L^{\mu}(X)$ belongs to

$$
\operatorname{Exp} L^{\mu}(X)=\left\{\exp B \exp (1+K) ; B \in L^{\mu \log }(X), K \in L^{-\infty}(X)\right\},
$$

if and only if $A$ is invertible (in particular, $A$ is elliptic) and $\Psi(A)=0$; in this sense generalizing the results of [19]. It turns out that $\bigcup_{\mu \in \mathbb{C}} \operatorname{Exp} L^{\mu}(X)$ is the group generated by the image of the exponential map (1.2) when $\mu$ runs through C. Note that $\Psi(A)=0$ means that $\sigma^{\mu}(A) \in S^{(\mu)}\left(\left(T^{*} X \times \Lambda\right) \backslash 0\right)$ possesses a continuous logarithm.

After reduction to holomorphic Mellin symbols in Section 2.3, Mellin symbols $G \in \mathcal{M}_{\mathcal{O}}^{\mu}(X)$ satisfying $\Psi\left(\left.G\right|_{\Gamma_{\beta}}\right)=0, \beta \in \mathbb{R}$, are factorized first. (Note that $\left.G\right|_{\Gamma_{\beta}} \in L^{\mu}\left(X ; \Gamma_{\beta}\right)$, where the line $\Gamma_{\beta}$ is identified with $\mathbb{R}$.) This is achieved by the basic formula (5.1) in which kernel cut-off enters, cf. Section 4.

The problem of constructing multiplicative decompositions of given meromorphic operator-valued functions is an older one. For such constructions in a general setup, particularly without regarding parameter-dependence, see [4] and the references therein.

The plan of the paper is as follows. In Section 2, we recall some facts from the theory of pseudodifferential operators and review the notion of a meromorphic Mellin symbol. Then, in Section 3, we define the exponential map (1.2) and characterize its image modulo smoothing operators, i.e., on the level of complete symbols. In Section 4, we recall the kernel cut-off technique which allows the construction of holomorphic Mellin symbols. Finally, in Section 5, we prove the main theorem.

Acknowledgement. In its main part, this work was done during a 10-month stay at the University of Bologna in 1998. I wish to express my gratitude to Pro- 
fessor O. Liess for his hospitality during that time and a lot of fruitful discussions. I also thank Professor B.-W. Schulze for bringing the problem on factorization to my knowledge.

Added in proof. Since the first version of this paper (Preprint 99/5, Institute of Mathematics, University of Potsdam) came out in 1999, a number of related work has appeared. So the proof has been outlined in the scalar-valued case (i.e., $\operatorname{dim} X=0)$ in $[18$, Sect. 2.1.3]. In [11, 12, 13], using also a result of [23], the factorization has been utilized to construct Mellin symbols obeying special properties.

\section{Prelimin ARIES}

In this section, we recall facts from pseudodifferential theory and introduce the class of meromorphic Mellin symbols. See, e.g., [7, 17, 20].

2.1 Parameter-dependent operators. To begin with, we remind of properties of parameter-dependent pseudodifferential operators.

2.1.1. Parameter-dependent symbols. Let $\Omega \subseteq \mathbb{R}^{n}$ be an open set and $\Lambda=\mathbb{R}^{l}$, $l \in \mathbb{N}$, be the parameter space. For $\bar{\mu} \in \mathbb{R}$, let $S_{1,0}^{\bar{\mu}}\left(\Omega \times \mathbb{R}^{n} \times \Lambda\right)$ denote the class of all functions $a(x, \xi, \lambda) \in C^{\infty}\left(\Omega \times \mathbb{R}^{n} \times \Lambda\right)$, where $x \in \Omega, \xi \in \mathbb{R}^{n}, \lambda \in \Lambda$, such that

$$
\left|\partial_{x}^{\alpha} \partial_{\xi}^{\beta} \partial_{\lambda}^{\gamma} a(x, \xi, \lambda)\right| \leq C_{\alpha \beta \gamma K}(1+|\xi|+|\lambda|)^{\bar{\mu}-|\beta|-|\gamma|}, \quad(x, \xi, \lambda) \in K \times \mathbb{R}^{n} \times \Lambda,
$$

holds for all compact subsets $K \subset \Omega$ and all multi-indices $\alpha, \beta \in \mathbb{N}^{n}, \gamma \in \mathbb{N}^{l}$, with certain constants $C_{\alpha \beta \gamma K}>0$. For $\mu \in \mathbb{C}$, let $S^{\mu}\left(\Omega \times \mathbb{R}^{n} \times \Lambda\right)$ denote the class of all functions $a(x, \xi, \lambda) \in S_{1,0}^{\bar{\mu}}\left(\Omega \times \mathbb{R}^{n} \times \Lambda\right), \bar{\mu}=\Re \mu$, possessing asymptotic expansions of the form

$$
a(x, \xi, \lambda) \sim \sum_{j=0}^{\infty} a_{(\mu-j)}(x, \xi, \lambda),
$$

where $a_{(\mu-j)}(x, \xi, \lambda) \in C^{\infty}\left(\Omega \times\left(\mathbb{R}^{N} \backslash\{0\}\right)\right)$ is positively homogenous of degree $\mu-j$ in $(\xi, \lambda)$. The expansion $(2.1)$ holds in the sense that

$$
a(x, \xi, \lambda)-\chi(\xi, \lambda) \sum_{k=0}^{j-1} a_{(\mu-k)}(x, \xi, \lambda) \in S_{1,0}^{\bar{\mu}-j}\left(\Omega \times \mathbb{R}^{n} \times \Lambda\right)
$$

is valid for any $j \in \mathbb{N}$, where $\chi \in C^{\infty}\left(\mathbb{R}^{n} \times \Lambda\right), \chi(\xi, \lambda)=0$ for $|\xi|+|\lambda| \leq 1 / 2$, and $\chi(\xi, \lambda)=1$ for $|\xi|+|\lambda| \geq 1$.

Let $L^{-\infty}(\Omega ; \Lambda)$ be the Schwartz space on $\Lambda$ with values in $L^{-\infty}(\Omega)$, where the latter is the space of all integral operators on $\Omega$ with $C^{\infty}$-kernel, and it is equipped with its natural Fréchet topology. For $\bar{\mu} \in \mathbb{R}$, let $L_{1,0}^{\bar{\mu}}(\Omega ; \Lambda)$ denote the 
class of all parameter-dependent pseudodifferential operators $A=A(\lambda)$ of order $\bar{\mu}$ (and type 1,0) with parameter space $\Lambda$, i.e., the class of all operators of the form

$$
A(\lambda)=\text { op }(a(x, \xi, \lambda))+C(\lambda)
$$

where

$$
\operatorname{op}(a(x, \xi, \lambda)) u=F_{\xi \rightarrow x}^{-1}\{a(x, \xi, \lambda) \hat{u}(\xi)\}, u \in C_{0}^{\infty}(\Omega),
$$

$a(x, \xi, \lambda) \in S_{1,0}^{\bar{\mu}}\left(\Omega \times \mathbb{R}^{n} \times \Lambda\right), F u(\xi)=\hat{u}(\xi)=\int_{\mathbb{R}^{n}} e^{-i x \xi} u(x) d x$ is the Fourier transform, and $C \in L^{-\infty}(\Omega, \Lambda)$. In particular, the operator convention op( ) = $\mathrm{op}_{x}(\mathrm{)})$ always refers to the spatial variable $x \in \Omega$ and its covariable $\xi \in \mathbb{R}^{n}$. For $\mu \in \mathbb{C}$, the class $L^{\mu}(\Omega ; \Lambda)$ of parameter-dependent classical pseudodifferential operators of order $\mu$ is analogously defined.

2.1.2. Operators on manifolds. Now let $X$ be a closed $C^{\infty}$-manifold, $\operatorname{dim} X=n$. For a sequentially continuous operator $A: C^{\infty}(X) \rightarrow \mathcal{D}^{\prime}(X)$ and a coordinate chart $\kappa: \Omega_{\kappa} \rightarrow \tilde{\Omega}_{\kappa}$, where $\Omega_{\kappa} \subset X$ and $\tilde{\Omega}_{\kappa} \subseteq \mathbb{R}^{n}$ are open subsets, $\kappa_{*} A$ denotes the operator push-forward of $A$ under the diffeomorphism $\kappa$, i.e., $\kappa_{*} A: C_{0}^{\infty}\left(\tilde{\Omega}_{\kappa}\right) \rightarrow$ $\mathcal{D}^{\prime}\left(\tilde{\Omega}_{\kappa}\right)$,

$$
\left(\kappa_{*} A\right) v=\kappa_{*} A\left(\kappa^{*} v\right), \quad v \in C_{0}^{\infty}\left(\tilde{\Omega}_{\kappa}\right)
$$

Definition 2.1. For $\mu \in \mathbb{C}$, the class $L^{\mu}(X ; \Lambda)$ consists of all parameter-dependent pseudodifferential operators $A=A(\lambda), \lambda \in \Lambda$, on $X$ such that $\kappa_{*} A \in$ $L^{\mu}\left(\tilde{\Omega}_{\kappa} ; \Lambda\right)$ for any coordinate chart $\kappa: \Omega_{\kappa} \rightarrow \tilde{\Omega}_{\kappa}$. For $\bar{\mu} \in \mathbb{R}$, the class $L_{1,0}^{\bar{\mu}}(X ; \Lambda)$ is analogously defined.

Remark 2.2. In Definition 2.1, it suffices to require $\left(\kappa_{i}\right)_{*} A \in L^{\mu}\left(\tilde{\Omega}_{i} ; \Lambda\right)$ for $i=$ $1, \ldots, m$, where $\left\{\Omega_{i}\right\}_{i=1}^{m}$ is a covering of $X$ by coordinate neighbourhoods and $\kappa_{i}: \Omega_{i} \rightarrow \tilde{\Omega}_{i}$ are the corresponding diffeomorphisms if it is additionally asked that the kernel of $A=A(\lambda)$ (with respect to a fixed positive $C^{\infty}$-density on $X$ ) belongs to $\mathcal{S}\left(\Lambda ; C^{\infty}((X \times X) \backslash \Delta)\right), \Delta=\{(x, x) \in X \times X ; x \in X\}$.

It is well-known that $\bigcup_{\bar{\mu} \in \mathbb{R}} L_{1,0}^{\bar{\mu}}(X ; \Lambda)$ is a filtered algebra under the composition of pseudodifferential operators on $X$.

In the sequel, we shall fix a Riemannian metric $g$ on $X$. We define the norm in $H^{0}(X)=L^{2}(X)$ with the help of the smooth density $|\operatorname{det} g(x)|^{1 / 2} d x$. Further we introduce the Sobolev space $H^{s}(X ; \Lambda), s \in \mathbb{R}$, as the space of all functions $u(x, \lambda)$ in $H^{s}(X)$ depending on the parameter $\lambda \in \Lambda$ such that the norm

$$
\|u\|_{H^{s}(X ; \Lambda)}=\left\{\int_{\Lambda}\left\|\left(1+|\lambda|^{2}-\Delta_{g}\right)^{s / 2} u\right\|_{H^{0}(X)}^{2} d \lambda\right\}^{1 / 2}
$$

is finite. Here $\Delta_{g}$ is the Laplace-Beltrami operator with respect to $g$. Then

$$
L_{1,0}^{\bar{\mu}}(X ; \Lambda) \subset \bigcap_{s \in \mathbb{R}} \mathcal{L}\left(H^{s}(X ; \Lambda), H^{s-\bar{\mu}}(X ; \Lambda)\right) .
$$


An operator $A \in L^{\mu}(X ; \Lambda)$ possesses a principal symbol $\sigma^{\mu}(A)(x, \xi, \lambda)$ that is invariantly defined on the total space of the bundle $\left(T^{*} X \times \Lambda\right) \backslash 0 \rightarrow X$,

$$
\sigma^{\mu}(A) \in S^{(\mu)}\left(\left(T^{*} X \times \Lambda\right) \backslash 0\right),
$$

where $S^{(\mu)}\left(\left(T^{*} X \times \Lambda\right) \backslash 0\right)$ is the space of all $C^{\infty}$-functions on $\left(T^{*} X \times \Lambda\right) \backslash 0$ that are positively homogeneous of degree $\mu$ in the fibres. Furthermore, there is the short exact split sequence

$$
0 \rightarrow L^{\mu-1}(X ; \Lambda) \rightarrow L^{\mu}(X ; \Lambda) \stackrel{\sigma^{\mu}}{\rightarrow} S^{(\mu)}\left(\left(T^{*} X \times \Lambda\right) \backslash 0\right) \rightarrow 0 .
$$

An operator $A \in L^{\mu}(X ; \Lambda)$ is called elliptic (more precisely, parameter-dependent elliptic) if $\sigma^{\mu}(A)(\zeta, \lambda) \neq 0$ for all $\zeta \in T^{*} X, \lambda \in \Lambda$, and $(\zeta, \lambda) \neq 0$.

Lemma 2.3. Let $A \in L^{\mu}(X ; \Lambda)$ be elliptic. Then $A$ is invertible if and only if $A(\lambda) \in L^{\mu}(X)$ is invertible for all $\lambda \in \Lambda$.

Proof. If $A \in L^{\mu}(X ; \Lambda)$ is elliptic, then there is a $\lambda_{0}>0$ such that $A(\lambda) \in L^{\mu}(X)$ is invertible for all $|\lambda| \geq \lambda_{0}$, cf. [20]. From this observation the assertion follows.

From Seeley's result [19] we infer:

Proposition 2.4. For $\mu \in \mathbb{C}$, we have

$$
\left(1+|\lambda|^{2}-\Delta_{g}\right)^{\mu / 2} \in L^{\mu}(X ; \Lambda),
$$

where the operator $\left(1+|\lambda|^{2}-\Delta_{g}\right)^{\mu / 2}$ is defined by means of the spectral theorem. The principal symbol of $\left(1+|\lambda|^{2}-\Delta_{g}\right)^{\mu / 2}$ is $\left(|\zeta|_{g}^{2}+|\lambda|^{2}\right)^{\mu / 2}$.

Eventually note that the spaces $L^{\mu}(X ; \Lambda), L_{1,0}^{\bar{\mu}}(X ; \Lambda)$ are Fréchet spaces in a natural way. Thereby, the topology of $L^{\mu}(X ; \Lambda)$ takes into account the sequence of the homogenous components of complete symbols in local coordinates, i.e.,

$$
L^{\mu}(X ; \Lambda)=\underset{j \rightarrow \infty}{\operatorname{proj} \lim } \bigoplus_{k<j} S^{(\mu-k)}\left(\left(T^{*} X \times \Lambda\right) \backslash 0\right) \oplus L_{1,0}^{\bar{\mu}-j}(X ; \Lambda)
$$

under the natural injections

$$
\begin{aligned}
& \bigoplus_{k \leq j} S^{(\mu-k)}\left(\left(T^{*} X \times \Lambda\right) \backslash 0\right) \oplus L_{1,0}^{\bar{\mu}-j-1}(X ; \Lambda) \\
& \quad \rightarrow \bigoplus_{k<j} S^{(\mu-k)}\left(\left(T^{*} X \times \Lambda\right) \backslash 0\right) \oplus L_{1,0}^{\bar{\mu}-j}(X ; \Lambda) \\
& \left(a_{(\mu)}, a_{(\mu-1)}, \ldots, a_{(\mu-j)}, A_{\mu-j-1}\right) \\
& \mapsto\left(a_{(\mu)}, a_{(\mu-1)}, \ldots, a_{(\mu-j+1)}, \varrho^{\mu-j}\left(a_{(\mu-j)}\right)+A_{\mu-j-1}\right),
\end{aligned}
$$


where $\varrho^{\mu-j}: S^{(\mu-j)}\left(\left(T^{*} X \times \Lambda\right) \backslash 0\right) \rightarrow L^{\mu-j}(X ; \Lambda)$ is a splitting of (2.2) (with $\mu$ replaced with $\mu-j)$, i.e., $\sigma^{\mu-j} \varrho^{\mu-j}=\operatorname{id}_{S_{(\mu-j)}\left(\left(T^{*} X \times \Lambda\right) \backslash 0\right)}$. In (2.3), the topology of $S^{(\mu-k)}\left(\left(T^{*} X \times \Lambda\right) \backslash 0\right)$ is the $C^{\infty}$-topology of functions on the sphere bundle of $\left(T^{*} X \times \Lambda\right) \backslash 0 \rightarrow X$. In particular, $L^{\mu}(X ; \Lambda)$ is a nuclear Fréchet space and $L^{-\infty}(X ; \Lambda)$ is its closed subspace.

2.1.3. Complete symbols. For further use, we recall the description of the algebra $\bigcup_{\mu \in \mathbb{C}} L^{\mu}(X ; \Lambda) / L^{-\infty}(X ; \Lambda)$ of complete symbols.

For $A \in L^{\mu}(X ; \Lambda)$, in local coordinates $\kappa: \Omega_{\kappa} \rightarrow \tilde{\Omega}_{\kappa}$ we have

$$
\kappa_{*} A=\mathrm{op}\left(a_{\kappa}(x, \xi, \lambda)\right)+C
$$

where $a_{\kappa} \in S^{\mu}\left(\tilde{\Omega}_{\kappa} \times \mathbb{R}^{n} \times \Lambda\right)$ and $C \in L^{-\infty}\left(\tilde{\Omega}_{\kappa} ; \Lambda\right)$. Here the symbol $a_{\kappa}$ is uniquely determined modulo $S^{-\infty}\left(\tilde{\Omega}_{\kappa} \times \mathbb{R}^{n} \times \Lambda\right)$. In particular, if

$$
a_{\kappa}(x, \xi, \lambda) \sim \sum_{j=0}^{\infty} a_{\kappa,(\mu-j)}(x, \xi, \lambda)
$$

with $a_{\kappa,(\mu-j)}(x, \xi, \lambda)$ being positively homogeneous of degree $\mu-j$ in $(\xi, \lambda)$, then the functions $a_{\kappa,(\mu-j)} \in S^{(\mu-j)}\left(\tilde{\Omega}_{\kappa} \times\left(\left(\mathbb{R}^{n} \times \Lambda\right) \backslash 0\right)\right)$ are uniquely determined.

Furthermore, if $\kappa^{\prime}: \Omega_{\kappa^{\prime}} \rightarrow \tilde{\Omega}_{\kappa^{\prime}}$ are other local coordinates, then

$$
\sum_{j=0}^{\infty} a_{\kappa^{\prime},(\mu-j)}\left(\kappa_{1}(x), \eta, \lambda\right)=\sum_{j=0}^{\infty} \sum_{\alpha \in \mathbb{N}^{n}} \frac{1}{\alpha !}\left(\partial_{\xi}^{\alpha} a_{\kappa,(\mu-j)}\right)\left(x,{ }^{t} \kappa_{1}^{\prime}(x) \eta, \lambda\right) \Phi_{\alpha}(x, \eta)
$$

on $\kappa\left(\Omega_{\kappa} \cap \Omega_{\kappa^{\prime}}\right) \times \mathbb{R}^{n} \times \Lambda$, where $\kappa_{1}=\kappa^{\prime} \kappa^{-1}: \kappa\left(\tilde{\Omega}_{\kappa} \cap \Omega_{\kappa^{\prime}}\right) \rightarrow \kappa^{\prime}\left(\tilde{\Omega}_{\kappa} \cap \Omega_{\kappa^{\prime}}\right)$ and

$$
\Phi_{\alpha}(x, \eta)=\left.D_{y}^{\alpha}\left(e^{i\left(\kappa_{1}(y)-\kappa_{1}(x)-\kappa_{1}^{\prime}(x)(y-x)\right) \eta}\right)\right|_{y=x}
$$

is a polynomial in $\eta$ of degree at most $|\alpha| / 2$. (2.6) holds in the sense of equality of asymptotic sums, which especially means that the right-hand side has to be re-ordered according to homogeneity.

Conversely, if, for each coordinate chart $\kappa: \Omega_{\kappa} \rightarrow \tilde{\Omega}_{\kappa}$, there is given a sequence $\left\{a_{\kappa,(\mu-j)}(x, \xi, \lambda)\right\}_{j=0}^{\infty}$ such that $a_{\kappa,(\mu-j)} \in S^{(\mu-j)}\left(\tilde{\Omega}_{\kappa} \times\left(\left(\mathbb{R}^{n} \times \Lambda\right) \backslash 0\right)\right)$ for $j \in \mathbb{N}$ and (2.6) holds for all coordinate charts $\kappa, \kappa^{\prime}$, then there exists an operator $A \in$ $L^{\mu}(X ; \Lambda)$ with the properties $(2.4),(2.5)$ for any coordinate charts $\kappa$. This operator $A$ is uniquely determined modulo $L^{-\infty}(X ; \Lambda)$.

Remark 2.5. As in Remark 2.2, it suffices to know the complete symbol of $A \in$ $L^{\mu}(X ; \Lambda)$ in local coordinates $\kappa_{i}: \Omega_{i} \rightarrow \tilde{\Omega}_{i}, i=1, \ldots, m$, where $\left\{\Omega_{i}\right\}_{i=1}^{m}$ is a covering of $X$. 
The multiplication in $\bigcup_{\mu \in \mathbb{C}} L^{\mu}(X ; \Lambda) / L^{-\infty}(X ; \Lambda)$ can be expressed in local coordinates. Indeed, if $a_{\kappa}=\left\{a_{\kappa,(\mu-j)}(x, \xi, \lambda)\right\}_{j=0}^{\infty}$ and $b_{\kappa}=\left\{b_{\kappa,(\nu-k)}(x, \xi, \lambda)\right\}_{k=0}^{\infty}$ are the complete symbols (in local coordinates) of two operators $A \in L^{\mu}(X ; \Lambda)$ and $B \in L^{\nu}(X ; \Lambda)$, respectively, then the complete symbol $(a \sharp b)_{\kappa}$ of the composite $A B$ (in the same coordinates) is given by the Leibniz product,

$$
(a \sharp b)_{\kappa,(\mu+\nu-l)}(x, \xi, \lambda)=\sum_{|\alpha|+j+k=l} \frac{1}{\alpha !} \partial_{\xi}^{\alpha} a_{\kappa,(\mu-j)}(x, \xi, \lambda) D_{x}^{\alpha} b_{\kappa,(\nu-k)}(x, \xi, \lambda),
$$

$l=0,1,2, \ldots$

2.2 Meromorphic Mellin symbols. Meromorphic Mellin symbols were introduced by Schulze, cf. $[16,17]$. We recall the basic definitions.

Let $X$ be a closed $C^{\infty}$-manifold. The case $\operatorname{dim} X=0$ (in which $X$ is assumed to be a point) is not excluded from the considerations; however, certain modifications are needed. In the following, the lines $\Gamma_{\beta}=\{z \in \mathbb{C} ; \Re z=\beta\}$, $\beta \in \mathbb{R}$, in the complex Mellin plane identified with $\mathbb{R}$ figure as parameter space $\Lambda=\mathbb{R}^{1}$. Accordingly, we have the spaces $L^{\mu}\left(X ; \Gamma_{\beta}\right), L_{1,0}^{\bar{\mu}}\left(X ; \Gamma_{\beta}\right)$ of parameterdependent pseudodifferential operators. For $\operatorname{dim} X=0, L^{\mu}\left(X ; \Gamma_{\beta}\right)=S^{\mu}\left(\Gamma_{\beta}\right)$ and $L_{1,0}^{\bar{\mu}}\left(X ; \Gamma_{\beta}\right)=S_{1,0}^{\bar{\mu}}\left(\Gamma_{\beta}\right)$, respectively.

A discrete asymptotic type for Mellin symbols is a sequences $P=\left\{\left(p_{j}, m_{j}\right.\right.$, $\left.\left.L_{j}\right)\right\}_{j \in \mathbb{Z}}$ such that $p_{j} \in \mathbb{C}, \Re p_{j} \rightarrow \mp \infty$ as $j \rightarrow \pm \infty, m_{j} \in \mathbb{N}$, and $L_{j} \subseteq L^{-\infty}(X)$ is a finite-dimensional subspace of finite-rank operators. Writing $P=\left\{\left(p_{j}, m_{j}, L_{j}\right)\right\}_{j \in \mathbb{Z}}$ with index set $\mathbb{Z}$ has the meaning of at most countable for $j$, in particular, one or both of the conditions $\Re p_{j} \rightarrow \mp \infty$ as $j \rightarrow \pm \infty$ may be void. The set of all discrete asymptotic types for Mellin symbols is denoted by $\underline{\operatorname{As}}(X)$. For $\operatorname{dim} X=0$, the spaces $L_{j}$ disappear from the definition: As is the set of all sequences $P=$ $\left\{\left(p_{j}, m_{j}\right)\right\}_{j \in \mathbb{Z}}$ such that $p_{j} \in \mathbb{C}, \Re p_{j} \rightarrow \mp \infty$ as $j \rightarrow \pm \infty$, and $m_{j} \in \mathbb{N}$. We put $\pi_{\mathbb{C}} P=\bigcup_{j \in \mathbb{Z}}\left\{p_{j}\right\}$. The empty asymptotic type, $\mathcal{O}$, is characterized by $\pi_{\mathbb{C}} \mathcal{O}=\emptyset$.

Definition 2.6. Let $\mu \in \mathbb{C}, P \in \underline{\operatorname{As}}(X)$.

(a) $\mathcal{M}_{\mathcal{O}}^{\mu}(X)$ is the space of all functions $G(z) \in \mathcal{A}\left(\mathbb{C} ; L^{\mu}(X)\right)$ such that $\left.G\right|_{\Gamma_{\beta}} \in$ $L^{\mu}\left(X ; \Gamma_{\beta}\right)$ uniformly for $\beta \in\left[\beta_{0}, \beta_{1}\right]$ and all $\beta_{0}, \beta_{1} \in \mathbb{R}, \beta_{0}<\beta_{1}$;

(b) $\mathcal{M}_{P}^{-\infty}(X)$ is the space of all functions $G(z) \in \mathcal{A}\left(\mathbb{C} \backslash \pi_{\mathbb{C}} P ; L^{-\infty}(X)\right)$ such that

(i) $G(z)$ is meromorphic on $\mathbb{C}$ with poles at $z=p_{j}$ of order $m_{j}+1$. Moreover, the principal part of the Laurent expansion of $G(z)$ at $z=p_{j}$ is $\sum_{k=0}^{m_{j}} G_{j k}\left(z-p_{j}\right)^{-1}$, where $G_{j k} \in L_{j}$ for $0 \leq k \leq m_{j}$

(ii) for every function $\chi \in C^{\infty}(\mathbb{C})$ with $\chi(z)=0$ in a neighbourhood of $\pi_{\mathbb{C}} P$ and $\chi(z)=1$ for $\operatorname{dist}\left(z, \pi_{\mathbb{C}} P\right) \geq 1$, we have $\left.\chi(z) G(z)\right|_{\Gamma_{\beta}} \in L^{-\infty}\left(X ; \Gamma_{\beta}\right)$ uniformly for $\beta \in\left[\beta_{0}, \beta_{1}\right]$ and all $\beta_{0}, \beta_{1} \in \mathbb{R}, \beta_{0}<\beta_{1}$;

(c) $\mathcal{M}_{P}^{\mu}(X)=\mathcal{M}_{\mathcal{O}}^{\mu}(X)+\mathcal{M}_{P}^{-\infty}(X)$.

For $\operatorname{dim} X=0$, we write $\mathcal{M}_{\mathcal{O}}^{\mu}, \mathcal{M}_{P}^{-\infty}$, and $\mathcal{M}_{P}^{\mu}$, respectively. 
$\mathcal{M}_{\mathcal{O}}^{\mu}(X), \mathcal{M}_{P}^{-\infty}(X)$ are nuclear Fréchet spaces in a natural manner, $\mathcal{M}_{P}^{\mu}(X)$ is then equipped with the topology of the non-direct sum.

Lemma 2.7. Let $G \in \mathcal{M}_{P}^{\mu}(X)$. Write $G=G_{0}+H$, where $G_{0} \in \mathcal{M}_{\mathcal{O}}^{\mu}(X), H \in$ $\mathcal{M}_{P}^{-\infty}(X)$. Then the principal symbol of $\left.G_{0}\right|_{\Gamma_{\beta}} \in L^{\mu}\left(X ; \Gamma_{\beta}\right)$ is independent of the chosen decomposition of $G$ and also independent of $\beta$.

Corollary 2.8. Let $G \in \mathcal{M}_{P}^{\mu}(X)$ be as in the previous lemma. Suppose that $\left.G_{0}\right|_{\Gamma_{\beta}} \in L^{-\infty}\left(X ; \Gamma_{\beta}\right)$ for some $\beta \in \mathbb{R}$. Then $G \in \mathcal{M}_{P}^{-\infty}(X)$.

Definition 2.9. Let $\mu \in \mathbb{C}, P \in \underline{\operatorname{As}}(X)$, and $G \in \mathcal{M}_{P}^{\mu}(X)$. Then $G$ is called (parameter-dependent) elliptic if its principal symbol $\sigma^{\mu}\left(\left.G_{0}\right|_{\Gamma_{\beta}}\right) \in S^{(\mu)}\left(\left(T^{*} X \times\right.\right.$ $\mathbb{R}) \backslash 0$ ) defined according to Lemma 2.7 is elliptic.

For the next result clarifying the significance of parameter-dependent ellipticity, see [17, Theorem 1.2.45]. (See also the proof of Lemma 2.3.)

Proposition 2.10. Let $\mu \in \mathbb{C}, P \in \underline{\mathrm{As}}(X)$, and $G \in \mathcal{M}_{P}^{\mu}(X)$ be elliptic. Then $G^{-1}(z) \in \mathcal{M}_{Q}^{-\mu}(X)$ for some resulting $Q \in \underline{\operatorname{As}}(X)$.

Notation 2.11. (a) For $\bar{\mu} \in \mathbb{R}$, we introduce the space $\mathcal{N}_{\mathcal{O}}^{\bar{\mu}}(X)$ as in Definition $2.6(\mathrm{a})$, with $L^{\mu}(X), L^{\mu}\left(X ; \Gamma_{\beta}\right)$ replaced with $L_{1,0}^{\bar{\mu}}(X), L_{1,0}^{\bar{\mu}}\left(X ; \Gamma_{\beta}\right)$.

(b) Similarly, for an open set $\Omega \subseteq \mathbb{R}^{n}$, we introduce the space $\mathcal{M}_{\mathcal{O}}^{\mu}(\Omega)$, where $\mu \in \mathbb{C}($ or $\mu=-\infty)$, as in Definition 2.6 (a), with $L^{\mu}(X), L^{\mu}\left(X ; \Gamma_{\beta}\right)$ replaced with $L^{\mu}(\Omega), L^{\mu}\left(\Omega ; \Gamma_{\beta}\right)$. For $G \in \mathcal{M}_{\mathcal{O}}^{\mu}(\Omega)$, we have

$$
G(z)=o p(g(x, \xi, z))+H(z)
$$

where $g \in S^{\mu}\left(\Omega \times \mathbb{R}^{n} \times \mathbb{C}_{\text {hol }}\right)$ and $H \in \mathcal{M}_{\mathcal{O}}^{-\infty}(\Omega)$. Here the space $S^{\mu}\left(\Omega \times \mathbb{R}^{n} \times \mathbb{C}_{\text {hol }}\right)$ consists of all $g \in \mathcal{A}\left(\mathbb{C} ; S^{\mu}\left(\Omega \times \mathbb{R}^{n}\right)\right)$ such that $\left.g\right|_{\Gamma_{\beta}} \in S^{\mu}\left(\Omega \times \mathbb{R}^{n} \times \Gamma_{\beta}\right)$ uniformly for $\beta \in\left[\beta_{0}, \beta_{1}\right]$ and all $\beta_{0}, \beta_{1} \in \mathbb{R}, \beta_{0}<\beta_{1}$.

2.3. Reduction to holomorphic Mellin symbols. First let us note that, for $G \in \mathcal{M}_{P}^{\mu}(X)$, the three representations

$$
G=F(1+H)=\left(1+H_{1}\right) F=F+H_{2},
$$

where $F \in \mathcal{M}_{\mathcal{O}}^{\mu}(X), F^{-1} \in \mathcal{M}_{\mathcal{O}}^{-\mu}(X)$ and $H \in \mathcal{M}_{Q}^{-\infty}(X), H_{1} \in \mathcal{M}_{Q_{1}}^{-\infty}(X)$, $H_{2} \in \mathcal{M}_{Q_{2}}^{-\infty}(X)$ for suitable $Q, Q_{1}, Q_{2} \in \underline{\operatorname{As}}(X)$, are equivalent. In fact, if one of the symbols $H, H_{1}, H_{2}$ is a Mellin symbol of order $-\infty$, then the other two are, due to the relations $F H=H_{1} F=H_{2}$ which may be multiplied by $F^{-1}$ from both sides. Moreover, if $P=\mathcal{O}$, then $Q=Q_{1}=Q_{2}=\mathcal{O}$.

By the same reason, in proving Theorem 1.1 it is sufficient to treat the case $P=\mathcal{O}$, as pronounced in the introduction. Suppose that the proof for holomorphic 
Mellin symbols has already been supplied. Let $G \in \mathcal{M}_{P}^{\mu}(X)$ as in Theorem 1.1. By Definition 2.6 (c), write $G=G_{0}+H_{1}$, where $G_{0} \in \mathcal{M}_{\mathcal{O}}^{\mu}(X), H_{1} \in \mathcal{M}_{P}^{-\infty}(X)$. In particular, $G_{0}$ is elliptic. Hence we can write $G_{0}=F\left(1+H_{0}\right)$, where $F \in \mathcal{M}_{\mathcal{O}}^{\mu}(X)$, $F^{-1} \in \mathcal{M}_{\mathcal{O}}^{-\mu}(X)$, and $H_{0} \in \mathcal{M}_{\mathcal{O}}^{-\infty}(X)$. Therefore,

$$
G=F\left(1+H_{0}\right)+H_{1}=F(1+H)
$$

where $H=H_{0}+F^{-1} H_{1} \in \mathcal{M}_{Q}^{-\infty}(X)$ for a suitable $Q \in \underline{\operatorname{As}}(X)$.

Below we shall restrict the considerations to holomorphic Mellin symbols. Likewise, we will produce an additive decomposition.

Notation 2.12. For $\mu \in \mathbb{C}$, Ell $L^{\mu}(X ; \Lambda)$ and $\operatorname{Inv} L^{\mu}(X ; \Lambda)$ denote the sets of all elliptic and all invertible elements in $L^{\mu}(X ; \Lambda)$, respectively. Thereby, $A \in L^{\mu}(X ; \Lambda)$ is invertible if there is an $B \in L^{-\mu}(X ; \Lambda)$ such that $A B=B A=1$. The same notation applies to $\mathcal{M}_{\mathcal{O}}^{\mu}(X)$.

\section{LOGARITHMS OF PSEUDODIFFERENTIAL OPERATORS}

In this section we introduce the operator classes $L^{\mu \log }(X ; \Lambda)$ whose elements serve as generators of one-parameter groups of pseudodifferential operators, define the exponential map (1.2), and characterize its image modulo smoothing operators.

3.1. The classes $\mathbf{L}^{\mu \log }(\mathbf{X} ; \Lambda)$. Let $\Omega \subseteq \mathbb{R}^{n}$ be an open subset. For $\mu \in \mathbb{C}$, the symbol class $S^{\mu \log }\left(\Omega \times \mathbb{R}^{n} \times \Lambda\right)$ consists of all functions $b(x, \xi, \lambda)$ such that

$$
b(x, \xi, \lambda)=\frac{\mu}{2} \log \left(1+|\xi|^{2}+|\lambda|^{2}\right)+c(x, \xi, \lambda)
$$

for a certain $c \in S^{0}\left(\Omega \times \mathbb{R}^{n} \times \Lambda\right)$.

Lemma 3.1. For $b \in C^{\infty}\left(\Omega \times \mathbb{R}^{n} \times \Lambda\right)$, we consider the following three conditions: (a) $b(x, \xi, \lambda) \in S^{\mu \log }\left(\Omega \times \mathbb{R}^{n} \times \Lambda\right)$;

(b) $b(x, \xi, \lambda)=\log a(x, \xi, \lambda)$ for some $a \in S^{\mu}\left(\Omega \times \mathbb{R}^{n} \times \Lambda\right)$, where the symbol $a$ is elliptic and $a(x, \xi, \lambda) \neq 0$ for all $(x, \xi, \lambda) \in \Omega \times \mathbb{R}^{n} \times \Lambda$;

(c) $\nabla_{\xi, \lambda} b \in S^{-1}\left(\Omega \times \mathbb{R}^{n} \times \Lambda ; \mathbb{C}^{n+l}\right)$ and $\sigma^{0}\left(\xi \cdot \nabla_{\xi} b+\lambda \cdot \nabla_{\lambda} b\right)=\mu$.

Then (a), (b) are equivalent, and both imply (c). Furthermore, if $\Omega \subseteq \mathbb{R}^{n}$ is simply connected, then (c) also implies (a), (b).

Proof. (a), (b) are obviously equivalent and (a) implies (c). The connection between $(\mathrm{a}),(\mathrm{b})$ is provided by $a(x, \xi, \lambda)=\left(1+|\xi|^{2}+|\lambda|^{2}\right)^{\mu / 2} e^{c(x, \xi, \lambda)}$.

Now suppose that $\Omega \subseteq \mathbb{R}^{n}$ is simply connected. Let us show that then (c) implies (b). In this proof, we will write $\theta=(\xi, \lambda)$ and $N=n+l$. The symbol $\mu \log \langle\theta\rangle$ satisfies (c). Let $c(x, \theta)=b(x, \theta)-\mu \log \langle\theta\rangle$. We will show that $c \in S^{0}(\Omega \times$ $\left.\mathbb{R}^{N}\right)$. By hypothesis, $\nabla_{\theta} c \in S^{-1}\left(\Omega \times \mathbb{R}^{N} ; \mathbb{C}^{N}\right)$ and $\theta \cdot \nabla_{\theta} c \in S^{-1}\left(\Omega \times \mathbb{R}^{N}\right)$. 
For $1 \leq k \leq N$, we set

$$
\frac{\partial c}{\partial \theta_{k}}(x, \theta) \sim \sum_{j=1}^{\infty} c_{(-j)}^{k}(x, \theta)
$$

where $c_{(-j)}^{k}(x, \theta)$ is positively homogeneous of degree $-j$ in $\theta$. Further, for $j \geq 2$, we set

$$
d_{(1-j)}(x, \theta)=\frac{1}{1-j} \sum_{k=1}^{N} \theta_{k} c_{(-j)}^{k}(x, \theta) .
$$

Then $d_{(1-j)}(x, \theta)$ is positively homogeneous of degree $1-j$ in $\theta$, while

$$
\begin{aligned}
\frac{\partial d_{(1-j)}}{\partial \theta_{k}}(x, \theta) & =\frac{1}{1-j}\left(c_{(-j)}^{k}(x, \theta)+\sum_{h=1}^{N} \theta_{h} \frac{\partial c_{(-j)}^{h}}{\partial \theta_{k}}(x, \theta)\right) \\
& =\frac{1}{1-j}\left(c_{(-j)}^{k}(x, \theta)+\sum_{h=1}^{N} \theta_{h} \frac{\partial c_{(-j)}^{k}}{\partial \theta_{h}}(x, \theta)\right) \\
& =\frac{1}{1-j}\left(c_{(-j)}^{k}(x, \theta)-j c_{(-j)}^{k}(x, \theta)\right) \\
& =c_{(-j)}^{k}(x, \theta) .
\end{aligned}
$$

Similarly, the form $c_{(-1)}^{1}(x, \theta) d \theta_{1}+\cdots+c_{(-1)}^{N}(x, \theta) d \theta_{N}$ is exact. This is immediate for $N=1$ and $N \geq 3$. For $N=2$, we observe that the integrability condition is $\int_{|\theta|=1}\left\{-\theta_{2} c_{(-1)}^{1}(x, \theta)+\theta_{1} c_{(-1)}^{2}(x, \theta)\right\} d \theta=0$ is satisfied. In fact, we have $\tau \nabla_{\theta} c(x, \tau \theta) \rightarrow\left(c_{(-1)}^{1}(x, \theta), c_{(-1)}^{2}(x, \theta)\right)$ as $\tau \rightarrow \infty$ uniformly in $|\theta|=1$. Thus $\partial_{\phi} c(x, \tau \theta) \rightarrow-\theta_{2} c_{(-1)}^{1}(x, \theta)+\theta_{1} c_{(-1)}^{2}(x, \theta)$ as $\tau \rightarrow \infty$ uniformly in $|\theta|=1$, where $(r, \phi)$ with $\theta=r e^{i \phi}$ denote polar coordinates, and

$$
\int_{|\theta|=1}\left\{-\theta_{2} c_{-1}^{1}(x, \theta)+\theta_{1} c_{-1}^{2}(x, \theta)\right\} d \theta=\lim _{\tau \rightarrow \infty} \int_{0}^{2 \pi} \partial_{\phi} c(x, \tau \theta) d \phi=0 .
$$

Therefore, there is a function $d_{(0)}(x, \theta) \in C^{\infty}\left(\Omega \times\left(\mathbb{R}^{N} \backslash 0\right)\right)$ such that

$$
\frac{\partial d_{(0)}}{\partial \theta_{k}}(x, \theta)=c_{(-1)}^{k}(x, \theta)
$$

for all $1 \leq k \leq N$. The relation

$$
\sum_{k=1}^{N} \theta_{k} \frac{\partial d_{(0)}}{\partial \theta_{k}}(x, \theta)=\sum_{k=1}^{N} \theta_{k} c_{(-1)}^{k}(x, \theta)=0
$$


implies that $d_{(0)}(x, \theta)$ is positively homogeneous of degree 0 in $\theta$.

Choose a function $d \in S^{0}\left(\Omega \times \mathbb{R}^{N}\right)$ such that

$$
d(x, \theta) \sim \sum_{j=0}^{\infty} d_{(-j)}(x, \theta) .
$$

By construction, $\nabla_{\theta}(c(x, \theta)-d(x, \theta)) \in S^{-\infty}\left(\Omega \times \mathbb{R}^{N} ; \mathbb{C}^{N}\right)$. Define $e(x, \theta)$ by the line integral

$$
e(x, \theta)=\int_{0}^{\theta} \sum_{k=1}^{N} \frac{\partial(c-d)}{\partial \theta_{k}}(x, \vartheta) d \vartheta_{k}
$$

Since

$$
\lim _{\tau \rightarrow \infty} e(x, \tau \theta)=\int_{0}^{\infty}\left(\theta \cdot \nabla_{\theta}\right)(c-d)(x, \tau \theta) d \tau=-c(x, 0)+d(x, 0)
$$

for all $\theta \in \mathbb{R}^{N},|\theta|=1$, we have $e \in S^{0}\left(\Omega \times \mathbb{R}^{N}\right)$ with principal symbol $-c(x, 0)+$ $d(x, 0)$. Actually, $e(x, \theta)=\chi(\theta)(-c(x, 0)+d(x, 0))+\tilde{e}(x, \theta)$, where $\chi \in C^{\infty}\left(\mathbb{R}^{N}\right)$, $\chi(\theta)=0$ for $|\theta| \leq 1 / 2, \chi(\theta)=1$ for $|\theta| \geq 1$, and $\tilde{e} \in S^{-\infty}\left(\Omega \times \mathbb{R}^{N}\right)$. Furthermore, $\nabla_{\theta}(c-(d+e))=0$. Hence there is a function $f \in C^{\infty}(\Omega)$ such that

$$
c(x, \theta)=d(x, \theta)+e(x, \theta)+f(x) .
$$

Therefore, we have $c \in S^{0}\left(\Omega \times \mathbb{R}^{N}\right)$.

By $L^{\mu \log }(\Omega ; \Lambda)$ we shall denote the class of all parameter-dependent pseudodifferential operators $B=B(\lambda), \lambda \in \Lambda$, on $\Omega$ which are of the form

$$
B(\lambda)=\text { op }(b(x, \xi, \lambda))+C(\lambda)
$$

where $b \in S^{\mu \log }\left(\Omega \times \mathbb{R}^{n} \times \Lambda\right)$ and $C \in L^{-\infty}(\Omega ; \Lambda)$.

Definition 3.2. For $\mu \in \mathbb{C}$, the class $L^{\mu \log }(X ; \Lambda)$ consists of all parameter-dependent pseudodifferential operators $B=B(\lambda), \lambda \in \Lambda$, on $X$ such that $\kappa_{*} B \in$

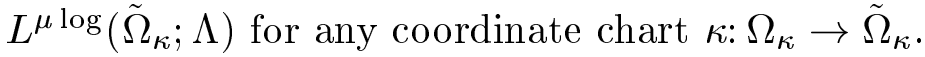

Remark 3.3. Remark 2.2 applies by analogy. That is, in Definition 3.2, it suffices to require $\left(\kappa_{i}\right)_{*} B \in L^{\mu \log }\left(\tilde{\Omega}_{i} ; \Lambda\right)$ for $i=1, \ldots, m$, where $\left\{\Omega_{i}\right\}_{i=1}^{m}$ is a covering of $X$ by coordinate neighbourhoods and $\kappa_{i}: \Omega_{i} \rightarrow \tilde{\Omega}_{i}$ are the corresponding diffeomorphisms if it is additionally asked that the kernel of $B=B(\lambda)$ (again with respect to a fixed positive $C^{\infty}$-density on $\left.X\right)$ belongs to $\mathcal{S}\left(\Lambda ; C^{\infty}((X \times X) \backslash \Delta)\right)$.

The next lemma is easily proved. Especially, it shows that $\bigcup_{\mu \in \mathbb{C}} L^{\mu \log }(X ; \Lambda)$ is a nuclear Fréchet space in an natural manner and, for a fixed $\mu \in \mathbb{C}, L^{\mu \log }(X ; \Lambda)$ is its closed, affine subspace. 
Lemma 3.4. $L^{\mu \log }(X ; \Lambda)$ consists of all operators $B$ of the form

$$
\frac{\mu}{2} \log \left(1+|\lambda|^{2}-\Delta_{g}\right)+C
$$

where the operator $\log \left(1+|\lambda|^{2}-\Delta_{g}\right)$ is defined by means of the spectral theorem and $C \in L^{0}(X ; \Lambda)$. Furthermore, if $g, \tilde{g}$ are Riemannian metrics on $X$, then

$$
\frac{\mu}{2} \log \left(1+|\lambda|^{2}-\Delta_{g}\right)-\frac{\mu}{2} \log \left(1+|\lambda|^{2}-\Delta_{\tilde{g}}\right) \in L^{0}(X ; \Lambda)
$$

and the principal symbol of this difference is

$$
\frac{\mu}{2} \log \left(|\zeta|_{g}^{2}+|\lambda|^{2}\right)-\frac{\mu}{2} \log \left(|\zeta|_{\tilde{g}}^{2}+|\lambda|^{2}\right)
$$

Lemma 3.4 yields that an operator $B \in L^{\mu \log }(X ; \Lambda)$ possesses a principal symbol $\sigma^{\mu \log (B)}$ that is invariantly defined on $\left(T^{*} X \times \Lambda\right) \backslash 0$ :

$$
\sigma^{\mu \log }(B)(\zeta, \lambda)=\frac{\mu}{2} \log \left(|\zeta|_{g}^{2}+|\lambda|^{2}\right)+\sigma^{0}(C)(\zeta, \lambda)
$$

where $B$ is given as in (3.2). Like in (2.2) we obtain a short exact split sequence

$$
0 \rightarrow L^{-1}(X ; \Lambda) \rightarrow L^{\mu \log }(X ; \Lambda) \stackrel{\sigma^{\mu \log }}{\longrightarrow} S^{(\mu \log )}\left(\left(T^{*} X \times \Lambda\right) \backslash 0\right) \rightarrow 0,
$$

where $S^{(\mu \log )}\left(\left(T^{*} X \times \Lambda\right) \backslash 0\right)$ is the space of all functions on $\left(T^{*} X \times \Lambda\right) \backslash 0$ of the form (3.3), with $\sigma^{0}(C)$ belonging to $S^{(0)}\left(\left(T^{*} X \times \Lambda\right) \backslash 0\right)$.

Remark 3.5. (a) We have $L^{\mu \log }(X ; \Lambda) \subset L_{1,0}^{\varepsilon}(X ; \Lambda)$ for any $\varepsilon>0$.

(b) Using the complete symbol of $C \in L^{0}(X ; \Lambda)$ in $(3.2)$, it is possible to introduce complete symbols for operators $B \in L^{\mu \log }(X ; \Lambda)$. We then obtain a space isomorphic to $L^{\mu \log }(X ; \Lambda) / L^{-\infty}(X ; \Lambda)$, cf. Section 2.1.3.

(c) It is seen that, for iterated commutators, we have

$$
\left[B_{1},\left[B_{2}, \ldots,\left[B_{j-1}, B_{j}\right] \ldots\right]\right] \in L^{-j+1}(X ; \Lambda)
$$

provided that $B_{k} \in L^{\mu_{k} \log }(X ; \Lambda)$ for $1 \leq k \leq j$ and $j \geq 2$, cf. also Lemma 3.1.

Definition 3.6. $\mathcal{M}_{\mathcal{O}}^{\mu \log }(X)$ consists of all functions $G(z) \in \mathcal{A}\left(\mathbb{C} ; L^{\mu \log }(X)\right)$ such that $\left.G\right|_{\Gamma_{\beta}} \in L^{\mu \log }\left(X ; \Gamma_{\beta}\right)$ uniformly for $\beta \in\left[\beta_{0}, \beta_{1}\right]$ and all $\beta_{0}, \beta_{1} \in \mathbb{R}, \beta_{0}<\beta_{1}$.

Remark 3.7. We again have that $\bigcup_{\mu \in \mathbb{C}} \mathcal{M}_{\mathcal{O}}^{\mu \log }(X)$ is a nuclear Fréchet space and, for a fixed $\mu \in \mathbb{C}, \mathcal{M}_{\mathcal{O}}^{\mu \log }(X)$ is its closed, affine subspace. For that notice that there is an element in $\mathcal{M}_{\mathcal{O}}^{\mu \log }(X)$ with principal symbol $(\mu / 2) \log \left(|\zeta|_{g}^{2}+|\Im z|^{2}\right)$, cf. 
Lemma 2.7, where the latter holds in an similar fashion for the spaces $\mathcal{M}_{\mathcal{O}}^{\mu \log }(X)$, and Section 4 below.

Notation 3.8. In the proof of Proposition 4.1, we will need the spaces $\mathcal{M}_{\mathcal{O}}^{\mu \log }(\Omega)$, for $\Omega \subseteq \mathbb{R}^{n}$ being open, which are defined in complete analogy to $\mathcal{M}_{\mathcal{O}}^{\mu}(\Omega)$, cf. Notation 2.11 (b). In particular, an arbitrary $G \in \mathcal{M}_{\mathcal{O}}^{\mu \log }(\Omega)$ has the form

$$
G(z)=\text { op }(g(x, \xi, z))+H(z),
$$

where $g \in S^{\mu \log }\left(\Omega \times \mathbb{R}^{n} \times \mathbb{C}_{\text {hol }}\right)$ and $H \in \mathcal{M}_{\mathcal{O}}^{-\infty}(\Omega)$. Here $S^{\mu \log }\left(\Omega \times \mathbb{R}^{n} \times \mathbb{C}_{\text {hol }}\right)$ is the space of all $b \in \mathcal{A}\left(\mathbb{C} ; S^{\mu \log }\left(\Omega \times \mathbb{R}^{n}\right)\right)$ such that $\left.b\right|_{\Gamma_{\beta}} \in S^{\mu \log }\left(\Omega \times \mathbb{R}^{n} \times \Gamma_{\beta}\right)$ uniformly for $\beta \in\left[\beta_{0}, \beta_{1}\right]$ and all $\beta_{0}, \beta_{1} \in \mathbb{R}, \beta_{0}<\beta_{1}$.

3.2. The exponential map. Now we define the exponential map (1.2).

We shall make widely use of the following strongly continuous family $U^{\mu}(t)$, $\mu \in \mathbb{C}$, of pseudodifferential operators on $X$, cf. Proposition 2.4 (recall that the Riemannian metric $g$ on $X$ is fixed):

$$
U^{\mu}(t)=\left(1+|\lambda|^{2}-\Delta_{g}\right)^{\mu t / 2} \in L^{\mu t}(X ; \Lambda), \quad t \in \mathbb{R} .
$$

Since here $\mu \in \mathbb{C}$ is arbitrary, we may actually allow $t \in \mathbb{C}$, and we obtain that, for each $u \in \mathcal{S}\left(\Lambda ; C^{\infty}(X)\right)$, the function $\mathbb{C} \rightarrow \mathcal{S}\left(\Lambda ; C^{\infty}(X)\right), t \mapsto U^{\mu}(t) u$ is holomorphic. Thus we get a holomorphic group on $\mathcal{S}\left(\Lambda ; C^{\infty}(X)\right)$. In particular,

$$
U^{\mu}(0)=1, \quad U^{\mu}(t) U^{\mu}\left(t^{\prime}\right)=U^{\mu}\left(t+t^{\prime}\right), \quad t, t^{\prime} \in \mathbb{C} .
$$

Let

$$
L_{\mu}=\frac{\mu}{2} \log \left(1+|\lambda|^{2}-\Delta_{g}\right) \in L^{\mu \log }(X ; \Lambda)
$$

be the generator of this group.

Lemma 3.9. For $\mu \in \mathbb{C}, \Re \mu \leq 0$, and $C \in L^{0}(X ; \Lambda), L_{\mu}+C$ is the generator of an analytic semigroup on $H^{0}(X ; \Lambda)$.

Proof. By Proposition 2.4, $L_{\mu}$ is the generator of an analytic semigroup on the space $H^{0}(X ; \Lambda)$ (i.e., we have $t \geq 0$ in (3.5)). It then also holds for $L_{\mu}+C$, since $C \in L^{0}(X ; \Lambda)$ is bounded on $H^{0}(X ; \Lambda)$.

For the moment, let $V(t)$ for $t \geq 0$ denote the semigroup generated by $L_{\mu}+C$ on $H^{0}(X ; \Lambda)$ provided that $\Re \mu \leq 0$. In case $\Re \mu \geq 0$, we obtain a semigroup $V(t)$ for $t \leq 0$ on the same space $H^{0}(X ; \Lambda)$.

There is another possibility to define $V(t)$. (In the following, the operator $C \in L^{0}(X ; \Lambda)$ is kept fixed.) Note that by bounded perturbation theory for strongly continuous semigroups, cf. [14, Section 3.1], the family $V(t)$ fulfils the integral equation

$$
V(t)=U^{\mu}(t)+\int_{0}^{t} U^{\mu}\left(t-t^{\prime}\right) C V\left(t^{\prime}\right) d t^{\prime}, \quad t \in \mathbb{R},
$$

on the half-line $t \Re \mu \leq 0$, where $V(t)$ is a priori defined. We are going to solve the Volterra-type integral equation (3.6) on the whole of $\mathbb{R}$. 
Proposition 3.10. There is a unique solution $V(t), t \in \mathbb{R}$, to Eq. (3.6) such that the function

$$
\mathbb{R} \rightarrow L^{0}(X ; \Lambda), \quad t \mapsto U^{\mu}(-t) V(t)
$$

is continuous. In particular, $V(t) \in L^{\mu t}(X ; \Lambda)$ for each $t \in \mathbb{R}$.

The proof is divided into three steps. First we argue on the level of complete symbols, i.e., we show that Eq. (3.6) can be solved modulo $C\left(\mathbb{R} ; L^{-\infty}(X ; \Lambda)\right)$.

Lemma 3.11. Let $u^{\mu}=u^{\mu}(t)$ be the complete symbol of the pseudodifferential operator family $U^{\mu}(t), t \in \mathbb{R}$, and $c$ be the complete symbol of $C \in L^{0}(X ; \Lambda)$. Then there is a unique solution $v=v(t)$ in complete symbols to the integral equation

$$
v(t)=u^{\mu}(t)+\int_{0}^{t} u^{\mu}\left(t-t^{\prime}\right) \sharp c \sharp v\left(t^{\prime}\right) d t^{\prime}, \quad t \in \mathbb{R},
$$

such that the function

$$
\mathbb{R} \rightarrow L^{0}(X ; \Lambda) / L^{-\infty}(X ; \Lambda), \quad t \mapsto u^{\mu}(-t) \sharp v(t)
$$

is continuous.

Proof. Let $\kappa: \Omega_{\kappa} \rightarrow \tilde{\Omega}_{\kappa}$ be a coordinate chart on $X$. Let $\left\{u_{(\mu t-j)}^{\mu}(t, x, \xi, \lambda)\right\}_{j=0}^{\infty}$ be the complete symbols of $U^{\mu}(t)$ in these coordinates. In particular, $u_{(\mu t-j)}^{\mu}(t, \cdot) \in$ $S^{(\mu t-j)}\left(\tilde{\Omega}_{\kappa} \times\left(\left(\mathbb{R}^{n} \times \Lambda\right) \backslash 0\right)\right)$ for each $t \in \mathbb{R}$. Similarly, let $\left\{c_{(-j)}(x, \xi, \lambda)\right\}_{j=0}^{\infty}$ be the complete symbols of $C$ in the same coordinates.

Written in components, Eq. (3.7) becomes

$$
\begin{aligned}
& v_{(\mu t-j)}(t, x, \xi, \lambda)=u_{(\mu t-j)}^{\mu}(t, \xi, \lambda) \\
& +\int_{0}^{t} u_{\left(\mu\left(t-t^{\prime}\right)\right)}^{\mu}\left(t-t^{\prime}, \xi, \lambda\right) c_{(0)}(x, \xi, \lambda) v_{\left(\mu t^{\prime}-j\right)}\left(t^{\prime}, x, \xi, \lambda\right) d t^{\prime} \\
& +\int_{0}^{t} \Pi_{j}\left(t, t^{\prime}, x, \xi, \lambda\right) d t^{\prime}
\end{aligned}
$$

for the complete symbol $\left\{v_{(\mu t-j)}(t, x, \xi, \lambda)\right\}_{j=0}^{\infty}$. Here $\Pi_{j}\left(t, t^{\prime}, x, \xi, \lambda\right)$ is positively homogeneous of degree $\mu t-j$ in $(\xi, \lambda)$ :

$$
\begin{aligned}
& \Pi_{j}\left(t, t^{\prime}, x, \xi, \lambda\right)=\sum_{\substack{|\alpha|+|\beta|+|\gamma|+\\
k+l+m=j, m<j}} \frac{1}{\alpha ! \beta ! \gamma !} \\
& \quad\left(\partial_{\xi}^{\alpha+\gamma} u_{\left(\mu\left(t-t^{\prime}\right)-k\right)}^{\mu}\right)\left(t-t^{\prime}, \xi, \lambda\right)\left(\partial_{x}^{\alpha} \partial_{\xi}^{\beta} c_{(-l)}\right)(x, \xi, \lambda)\left(\partial_{x}^{\beta+\gamma} v_{\left(\mu t^{\prime}-m\right)}\right)\left(t^{\prime}, x, \xi, \lambda\right) .
\end{aligned}
$$

The space $S^{(\mu t-j)}\left(\tilde{\Omega}_{\kappa} \times\left(\left(\mathbb{R}^{n} \times \Lambda\right) \backslash 0\right)\right)$ is isomorphic to $C^{\infty}\left(\tilde{\Omega}_{\kappa} \times S^{n+l-1}\right)$ whose topology can be given by sequence of submultiplicative seminorms. This allows 
to reduce solving the integral equation (3.7) to a series of corresponding integral equations in a projective system of Banach algebras (providing the topology of $\left.C^{\infty}\left(\tilde{\Omega}_{\kappa} \times S^{n+l-1}\right)\right)$ in which such Volterra-type integral equations are known to be uniquely solvable. (The complete argument is given in the proof of the next lemma.) Using this, Eq. (3.8) can be successively solved for $j=0,1,2, \ldots$; thus obtaining the complete symbol $v(t)=\left\{v_{(\mu t-j)}(t, x, \xi, \lambda)\right\}_{j=0}^{\infty}$.

Performing this construction for different coordinate charts $\kappa: \Omega_{\kappa} \rightarrow \tilde{\Omega}_{\kappa}$ and $\kappa^{\prime}: \Omega_{\kappa^{\prime}} \rightarrow \tilde{\Omega}_{\kappa^{\prime}}$, it is seen that the resulting $v_{\kappa}=v_{\kappa}(t)$ and $v_{\kappa^{\prime}}=v_{\kappa^{\prime}}(t)$ are compatible on the joint intersection $\Omega_{\kappa} \cap \Omega_{\kappa^{\prime}}$ in the sense of (2.6). Thus we have obtained a global solution $v=v(t)$ to Eq. (3.7).

To complete the proof of Proposition 3.10, we still need the following result.

Lemma 3.12. For each $F \in C\left(\mathbb{R} ; L^{-\infty}(X ; \Lambda)\right)$, there is a unique solution $W \in$ $C\left(\mathbb{R} ; L^{-\infty}(X ; \Lambda)\right)$ to the integral equation

$$
W(t)=F(t)+\int_{0}^{t} U^{\mu}\left(t-t^{\prime}\right) C W\left(t^{\prime}\right) d t^{\prime}, \quad t \in \mathbb{R} .
$$

Proof. It suffices to solve Eq. (3.9) on finite intervals $[-T, T], T>0$. For $k \in \mathbb{N}$, $G \in L^{-\infty}(X, \Lambda)$, we define

$$
\|G\|_{k}=\sum_{j \leq k}\left\|\partial_{\lambda}^{j} G\right\|_{H^{-k}(X ; \Lambda) \rightarrow H^{k}(X ; \Lambda)} .
$$

Then \|\|$_{k}, k \in \mathbb{N}$, is a fundamental seminorm system for the Fréchet topology of $L^{-\infty}(X ; \Lambda)$. Furthermore, for $j \leq k,|t| \leq T$,

$$
\left\|U^{\mu}(t) C G\right\|_{k} \leq M_{T, k}\|G\|_{k}
$$

for some constant $M_{T, k}>0$ depending on $T>0, k \in \mathbb{N}$, and $C \in L^{0}(X ; \Lambda)$, because of

$$
\left\|\partial_{\lambda}^{j}\left(U^{\mu}(t) C G\right)\right\|_{H^{-k} \rightarrow H^{k}} \leq \sum_{j_{0}+j_{1}=j}\left(\begin{array}{c}
j \\
j_{0}
\end{array}\right)\left\|\partial_{\lambda}^{j_{0}}\left(U^{\mu}(t) C\right) \partial_{\lambda}^{j_{1}} G\right\|_{H^{-k} \rightarrow H^{k}}
$$

and

$$
\begin{aligned}
& \left\|\partial_{\lambda}^{j_{0}}\left(U^{\mu}(t) C\right) \partial_{\lambda}^{j_{1}} G\right\|_{H^{-k} \rightarrow H^{k}} \\
& \leq \begin{cases}\left\|\partial_{\lambda}^{j_{0}}\left(U^{\mu}(t) C\right)\right\|_{H^{k+\bar{\mu} t-j_{0}} \rightarrow H^{k}}\left\|\partial_{\lambda}^{j_{1}} G\right\|_{H^{-k} \rightarrow H^{k+\bar{\mu} t-j_{0}}} & \text { if } \bar{\mu} t-j_{0} \geq 0, \\
\left\|\partial_{\lambda}^{j_{0}}\left(U^{\mu}(t) C\right)\right\|_{H^{k} \rightarrow H^{k-\bar{\mu} t+j_{0}}}\left\|\partial_{\lambda}^{j_{1}} G\right\|_{H^{-k} \rightarrow H^{k}} & \text { if } \bar{\mu} t-j_{0}<0,\end{cases}
\end{aligned}
$$

where $\bar{\mu}=\Re \mu$. 
Then Eq. (3.9) can be solved by iteration in the usual manner. We set $W_{0}(t)=$ $F(t),|t| \leq T$, and, for $j \geq 0$,

$$
W_{j+1}(t)=\int_{0}^{t} U^{\mu}\left(t-t^{\prime}\right) C W_{j}\left(t^{\prime}\right) d t^{\prime}, \quad|t| \leq T
$$

By induction on $j$, we get the estimate

$$
\left\|W_{j}(t)\right\|_{k} \leq \frac{F_{T, k}}{j !}\left(M_{k, T}|t|\right)^{j}, \quad|t| \leq T
$$

where $F_{T, k}=\sup _{|t| \leq T}\|F(t)\|_{k}$. Indeed, (3.10) is valid for $j=0$. Assuming that (3.10) has already be shown to hold for some $j$, we obtain

$$
\begin{aligned}
\left\|W_{j+1}(t)\right\|_{k} \leq \int_{0}^{|t|} & M_{T, k}\left\|W_{j}\left(t^{\prime}\right)\right\|_{k} d\left|t^{\prime}\right| \\
& \leq \int_{0}^{|t|} M_{T, k} \frac{F_{T, k}}{j !}\left(M_{k, T}\left|t^{\prime}\right|\right)^{j} d\left|t^{\prime}\right|=\frac{F_{T, k}}{(j+1) !}\left(M_{k, T}|t|\right)^{j+1}
\end{aligned}
$$

Therefore, the series $W=\sum_{j=0}^{\infty} W_{j}$ converges absolutely in the space $C([-T, T]$; $\left.L^{-\infty}(X ; \Lambda)\right),\|W(t)\|_{k} \leq F_{T, k} \exp \left(M_{k, T}|t|\right)$, and

$$
\begin{array}{r}
W(t)=F(t)+\sum_{j=0}^{\infty} W_{j+1}(t)=F(t)+\int_{0}^{t} U^{\mu}\left(t-t^{\prime}\right) C \sum_{j=0}^{\infty} W_{j}\left(t^{\prime}\right) d t^{\prime} \\
=F(t)+\int_{0}^{t} U^{\mu}\left(t-t^{\prime}\right) C W\left(t^{\prime}\right) d t^{\prime}
\end{array}
$$

Thus, $W$ is a solution of (3.9). If $\tilde{W} \in C\left([-T, T] ; L^{-\infty}(X ; \Lambda)\right)$ is another solution, then

$$
(W-\tilde{W})(t)=\int_{0}^{t} U^{\mu}\left(t-t^{\prime}\right) C(W-\tilde{W})\left(t^{\prime}\right) d t^{\prime}
$$

and estimating as above yields

$$
\|(W-\tilde{W})(t)\|_{k} \leq \frac{W_{T, k}}{j !}\left(M_{T, k}|t|\right)^{j}
$$

for $j=0,1,2, \ldots$, where $W_{T, k}=\sup _{|t| \leq T}\|(W-\tilde{W})(t)\|_{k}$. Letting $j \rightarrow \infty$, we get $(W-\tilde{W})(t)=0$ for all $|t| \leq T$.

Proof of Proposition 3.10. We choose a family $V^{\prime}(t), t \in \mathbb{R}$, of pseudodifferential operators possessing the complete symbol $v=v(t)$ constructed in Lemma 3.11 and 
such that the family $U^{\mu}(-t) V^{\prime}(t), t \in \mathbb{R}$, is continuous taking values in $L^{0}(X ; \Lambda)$. By construction

$$
V^{\prime}(t)=U^{\mu}(t)+\int_{0}^{t} U^{\mu}\left(t-t^{\prime}\right) C V^{\prime}\left(t^{\prime}\right) d t^{\prime}-F(t)
$$

for a certain family $F \in C\left(\mathbb{R} ; L^{-\infty}(X ; \Lambda)\right)$. Let $W \in C\left(\mathbb{R} ; L^{-\infty}(X ; \Lambda)\right)$ be the solution to Eq. (3.9), with the same $F$. Then $V=V^{\prime}+W$ is the desired solution to Eq. (3.6).

Uniqueness of this solution $V$ also follows, since any solution must have $v=v(t)$ constructed in Lemma 3.11 as complete symbol and, therefore, differs from $V$ by a function belonging to $C\left(\mathbb{R} ; L^{-\infty}(X ; \Lambda)\right)$. But then the uniqueness in Lemma 3.12 yields the assertion.

Remark 3.13. In case $\left[L_{\mu}, C\right]=0$, we simply have $V(t)=\exp \left(t L_{\mu}\right) \exp D(t)$, where

$$
D(t)=\int_{0}^{t} U^{\mu}\left(-t^{\prime}\right) C U^{\mu}\left(t^{\prime}\right) d t^{\prime}
$$

Definition 3.14. Let $B \in L^{\mu \log }(X ; \Lambda)$. Then the group $\exp (t B), t \in \mathbb{R}$, is the analytic group $V(t), t \in \mathbb{R}$, of pseudodifferential operators defined in Proposition 3.10, where $B=(\mu / 2) \log \left(1+|\lambda|^{2}-\Delta_{g}\right)+C$ and $C \in L^{0}(X ; \Lambda)$.

In particular, $\exp B=V(1) \in L^{\mu}(X ; \Lambda)$.

Lemma 3.15. For $B \in L^{\mu \log }(X ; \Lambda)$, we have

$$
\sigma^{\mu}(\exp B)=\exp \left(\sigma^{\mu \log }(B)\right)
$$

Proof. For $j=0$, Eq. (3.8) reads

$$
\begin{aligned}
v_{(\mu t)}(t, x, \xi, \lambda)=u_{(\mu t)}^{\mu} & (t, \xi, \lambda) \\
& +\int_{0}^{t} u_{\left(\mu\left(t-t^{\prime}\right)\right)}^{\mu}\left(t-t^{\prime}, \xi, \lambda\right) c_{(0)}(x, \xi, \lambda) v_{\left(\mu t^{\prime}\right)}\left(t^{\prime}, x, \xi, \lambda\right) d t^{\prime}
\end{aligned}
$$

The unique solution $v_{(\mu t)}(t, x, \xi, \lambda)$ to this equation is seen to be

$$
v_{(\mu t)}(t, x, \xi, \lambda)=\left(\sum_{i, j=1}^{n} g^{i j}(x) \xi_{i} \xi_{j}+|\lambda|^{2}\right)^{\mu t / 2} \exp \left(t c_{(0)}(x, \xi, \lambda)\right) .
$$

This yields the assertion.

Let $\operatorname{Exp} L^{0}(X ; \Lambda)$ be the group generated in $\operatorname{Inv} L^{0}(X ; \Lambda)$ by all elements of the form $\exp C$, where $C \in L^{0}(X ; \Lambda)$. Further let

$$
\operatorname{Exp} L^{\mu}(X ; \Lambda)=\left\{A \in L^{\mu}(X ; \Lambda) ; A=\left(\exp L_{\mu}\right) C, C \in \operatorname{Exp} L^{0}(X ; \Lambda)\right\} .
$$

We immediately get: 
Lemma 3.16. $\bigcup_{\mu \in \mathbb{C}} \operatorname{Exp} L^{\mu}(X ; \Lambda)$ is a normal divisor in $\bigcup_{\mu \in \mathbb{C}} \operatorname{Inv} L^{\mu}(X ; \Lambda)$. More precisely, if $A \in \operatorname{Exp} L^{\mu}(X ; \Lambda), A^{\prime} \in \operatorname{Exp} L^{\mu^{\prime}}(X ; \Lambda)$, then we have $A A^{\prime} \in$ $\operatorname{Exp} L^{\mu+\mu^{\prime}}(X ; \Lambda)$. If $B \in L^{\mu \log }(X ; \Lambda)$ and $D \in \operatorname{Inv} L^{\rho}(X ; \Lambda)$, then $D B D^{-1} \in$ $L^{\mu \log }(X ; \Lambda)$ and

$$
\exp \left(D B D^{-1}\right)=D(\exp B) D^{-1}
$$

Proof. The key is to prove (3.11). But if $B \in L^{\mu \log }(X ; \Lambda)$ and $D \in \operatorname{Inv} L^{\rho}(X ; \Lambda)$, then $D B D^{-1} \in L^{\mu \log }(X ; \Lambda)$ by Lemma 3.1 and, moreover, if $V(t)=\exp (t B)$, $t \in \mathbb{R}$, then $V(t)$ is the unique solution to $V^{\prime}(t)=B V(t), V(0)=I$ so that $D V(t) D^{-1}$ is the unique solution to $W^{\prime}(t)=\left(D B D^{-1}\right) W(t), W(0)=I$, and $\exp \left(t\left(D B D^{-1}\right)\right)=D \exp (t B) D^{-1}$.

Using (3.11) with $D=U^{\mu}(1)$, it follows that, for each $C \in \operatorname{Exp} L^{0}(X ; \Lambda)$, there is a $C^{\prime} \in \operatorname{Exp} L^{0}(X ; \Lambda)$ such that $C^{\prime} U^{\mu}(1)=U^{\mu}(1) C$. From that, then all the rest is concluded.

Remark 3.1\%. It can be shown that if $B=L_{\mu}+C \in L^{\mu \log }(X ; \Lambda), B^{\prime}=L_{\mu^{\prime}}+$ $C^{\prime} \in L^{\mu^{\prime} \log }(X ; \Lambda)$ such that $|\mu|,\left|\mu^{\prime}\right|$ are small enough and $C, C^{\prime} \in L^{0}(X ; \Lambda)$ are sufficiently small in $\mathcal{L}\left(H^{0}(X ; \Lambda)\right)$, then there is a $B^{\prime \prime} \in L^{\left(\mu+\mu^{\prime}\right) \log }(X ; \Lambda)$ such that

$$
\exp B \exp B^{\prime}=\exp B^{\prime \prime}
$$

Furthermore, $B^{\prime \prime}$ can be calculated from $B, B^{\prime}$ using the Campbell-Hausdorff formula. See, e.g., [6].

3.3 The topological invariant $\Psi(\mathbf{A})$. Next we are concerned with the characterization of the image of the exponential map (1.2). We start with a digression to topology.

Let $Y$ be a locally finite and countable $C W$-complex. Let $S^{1}=\{\zeta \in \mathbb{C} ;|\zeta|=$ $1\}$. We consider the collection $\left[Y, S^{1}\right]$ of all homotopy classes of continuous maps $f: Y \rightarrow S^{1}$. The group structure of $S^{1}$ converts $\left[Y, S^{1}\right]$ into an Abelian group.

Let $f: Y \rightarrow S^{1}$ be continuous. Then $f$ is homotopic to a $C W$-map, where we regard $S^{1}$ as a $C W$-complex with 0 -cell $\{1\}$ and 1-cell $S^{1} \backslash\{1\}$. That means that there is a continuous map $F:[0,1] \times Y \rightarrow S^{1}$ such that $F(0, \cdot)=f$ and $F(1, y)=1$ for all $y \in Y^{0}$. Obstruction theory (see $[20, \S 33]$ ) provides us with a difference element in $\mathcal{C}^{1}\left(Y ; \pi_{1}\left(S^{1}\right)\right)$, the group of 1-cochains of $Y$ with values in $\pi_{1}\left(S^{1}\right)$, whose vanishing determines the possibility of joining $F(1, \cdot)$ on $Y^{1}$ by homotopy to the constant map 1 ; thereby leaving $F(1, \cdot)$ invariant on $Y^{0}$. (See the next proof for an explicit description.) This difference element is actually a 1-cocycle, since $f$ and the constant map 1 are a priori defined on the whole of $Y$. Thus the difference element determines an element in $H^{1}(Y ; \mathbb{Z})$ which we denote by $\Lambda(f)$. Here we have identified $\pi_{1}\left(S^{1}\right) \cong \mathbb{Z}$ via the mapping degree. 
Lemma 3.18. The map so constructed induces an isomorphism of groups

$$
\left[Y, S^{1}\right] \rightarrow H^{1}(Y ; \mathbb{Z}),[f] \mapsto \Lambda(f) .
$$

Proof. Let $\sigma_{j}^{i}, j \in \mathcal{I}_{i}$, be the $i$-cells of $Y, Y^{i}=\bigcup_{i \in \mathcal{I}_{i}} \sigma_{j}^{i}$.

Let $f: Y \rightarrow S^{1}$ be a $C W$-map. Let $\gamma:[0,1] \rightarrow Y$ be the characteristic map of the 1-cell $\sigma_{j}^{1}$, where $\partial \sigma_{j}^{1}=\sigma_{j_{1}}^{0}-\sigma_{j_{0}}^{0}$. Since $f\left(\sigma_{j_{0}}^{0}\right)=f\left(\sigma_{j_{1}}^{0}\right)=1$, the map $f \gamma:[0,1] \rightarrow S^{1}$ descends to a map $S^{1} \rightarrow S^{1}$. Denote the mapping degree of the resulting map $S^{1} \rightarrow S^{1}$ by $\alpha_{j}^{f}$. Then $\Lambda(f)$ is the class of the 1-cocycle $\sigma_{j}^{1} \mapsto \alpha_{j}^{f}$, $j \in \mathcal{I}_{1}$.

That, now for arbitrary $f: Y \rightarrow S^{1}$, the map $f \mapsto \Lambda(f)$ is correctly defined is implied by the fact that if $f_{0}, f_{1}: Y \rightarrow S^{1}$ are $C W$-maps, where $f_{0}$ is homotopic to $f_{1}$, then $\alpha_{j}^{f_{0}}=\alpha_{j}^{f_{1}}$ holds for all $j \in \mathcal{I}_{1}$. This also shows that $\Lambda(f)$ only depends on the homotopy class $[f]$ and that the assignment $[f] \mapsto \Lambda(f)$ is indeed a group homomorphism $\left[Y, S^{1}\right] \rightarrow H^{1}(Y ; \mathbb{Z})$.

We verify that (3.12) is injective. Let $f: Y \rightarrow S^{1}$ be a $C W$-map such that $\Lambda(f)=0$, i.e., $\alpha_{j}^{f}=0$ for all $j \in \mathcal{I}_{1}$. This means that $f$ can be joined on $Y^{1}$ by homotopy to the constant map 1, leaving $f$ invariant on $Y^{0}$. Since $\pi_{i}\left(S^{1}\right)=0$ for $i \geq 2$, all further obstacles in extending this homotopy to $Y^{i}, i \geq 2$, and thus to $Y$ vanish. Hence $f$ and the constant map 1 are homotopic, i.e., $[f]=0$ in $\left[Y, S^{1}\right]$.

Eventually, we show that (3.12) is surjective. Choose a class in $H^{1}(Y ; \mathbb{Z})$ and let this class be represented by the 1-cocycle $\sigma_{j}^{1} \mapsto \alpha_{j}$, where $\alpha_{j} \in \mathbb{Z}$. Choose $q \in Y^{0}$ arbitrarily and set $f(q)=e^{i 0}=1$. Define $f(y) \in S^{1}$ for $y \in Y^{0} \cup Y^{1}$ just by increasing the argument of $f(y)$ along $\sigma_{j}^{1}$ by the value $2 \pi \alpha_{j}$. If $\sigma=\sum_{k \in \mathcal{I}_{1}} \beta_{k} \sigma_{k}^{1}$, $\beta_{k} \in \mathbb{Z}$, is a 1-chain with $\partial \sigma=\sigma_{j}^{0}-q$, then $\arg f\left(\sigma_{j}^{0}\right)=2 \pi \sum \alpha_{k} \beta_{k}$ depends on $\sigma_{j}^{0}$, but is independent of $\sigma$. This shows that $f$ can be correctly defined on $Y^{0} \cup Y^{1}$ in this way. By construction, the obstruction to extend $f$ to $Y^{2}$, which belongs to $\mathcal{C}^{2}\left(Y ; \pi_{1}\left(S^{1}\right)\right)$, vanishes. All other obstructions to extend $f$ to higher skeletons $Y^{i}, i \geq 3$, vanish for trivial reason; thus we can extend $f$ to all of $Y$. Obviously, $\alpha_{j}^{f}=\alpha_{j}$ for all $j \in \mathcal{I}_{1}$. Hence (3.12) is surjective.

Remark 3.19. (a) For a continuous map $f: Y \rightarrow \mathbb{C} \backslash\{0\}$, there exists a continuous logarithm (i.e., there is a continuous map $g: Y \rightarrow \mathbb{C}$ such that $\exp g=f$ ) if and only if $[f /|f|]=[1]=0$ in $\left[Y, S^{1}\right]$. By the above construction, this holds if and only if $\Lambda(f /|f|)=0$.

(b) There is still an alternative description of the isomorphism (3.12). By the universal coefficient theorem,

$$
\operatorname{Hom}\left(H_{1}(Y) ; \mathbb{Z}\right) \cong H^{1}(Y ; \mathbb{Z}) .
$$

Under this identification $\Lambda(f)=f_{*}$, where $f_{*}: H_{1}(Y) \rightarrow H_{1}\left(S^{1}\right) \cong \mathbb{Z}$.

Now we turn to the definition of the invariant $\Psi(A)$ for $A \in \operatorname{Ell} L^{\mu}(X ; \Lambda)$. Recall that $A \in \operatorname{Ell} L^{\mu}(X ; \Lambda)$ means that $\sigma^{\mu}(A)(\zeta, \lambda) \neq 0$ for all $(\zeta, \lambda) \in\left(T^{*} X \times \Lambda\right) \backslash 0$. 
Definition 3.20. For $A \in \operatorname{Ell} L^{\mu}(X ; \Lambda)$, we set

$$
\Psi(A)=\Lambda\left(\frac{\sigma^{\mu}(A)}{\left|\sigma^{\mu}(A)\right|}\right) \in H^{1}\left(\left(T^{*} X \times \Lambda\right) \backslash 0 ; \mathbb{Z}\right) .
$$

Lemma 3.21. For $A \in \operatorname{Ell} L^{\mu}(X ; \Lambda), A^{\prime} \in \operatorname{Ell} L^{\mu^{\prime}}(X ; \Lambda)$,

$$
\Psi\left(A A^{\prime}\right)=\Psi(A)+\Psi\left(A^{\prime}\right) .
$$

Proof. This follows from $\sigma^{\mu+\mu^{\prime}}\left(A A^{\prime}\right)=\sigma^{\mu}(A) \sigma^{\mu^{\prime}}\left(A^{\prime}\right)$.

Remark 3.22. (a) Let $\Lambda=\Lambda_{1} \times \Lambda_{2}$ be a splitting of the parameter space, i.e., $\mathbb{R}^{l}=\mathbb{R}^{l_{1}} \oplus \mathbb{R}^{l_{2}}$, where $l=l_{1}+l_{2}$. Then, for $A \in$ Ell $L^{\mu}(X ; \Lambda)$ and fixed $\lambda_{2} \in \Lambda_{2}$, we have $A\left(\cdot, \lambda_{2}\right) \in \operatorname{Ell} L^{\mu}\left(X ; \Lambda_{1}\right)$ and

$$
\iota^{*} \Psi(A)=\Psi\left(A\left(\cdot, \lambda_{2}\right)\right),
$$

where $\iota$ : $\left(T^{*} X \times \Lambda_{1}\right) \backslash 0 \rightarrow\left(T^{*} X \times \Lambda\right) \backslash 0$ is defined by $\left(\zeta, \lambda_{1}\right) \mapsto\left(\zeta, \lambda_{1}, 0\right)$.

(b) For $A \in \operatorname{Ell} L^{\mu}(X)$ and $\Psi(A)=0$, we have ind $A=0$, where ind $A$ is the index of $A$ as Fredholm operator in $\mathcal{L}\left(H^{s}(X), H^{s-\bar{\mu}}(X)\right), s \in \mathbb{R}, \bar{\mu}=\Re \mu$.

Notation 3.23. For $G \in \mathcal{M}_{\mathcal{O}}^{\mu}(X), \Psi(G)$ denotes the $\Psi$-invariant of the principal symbol of $\left.G\right|_{\Gamma_{\beta}} \in L^{\mu}\left(X ; \Gamma_{\beta}\right)$ that is indeed independent of $\beta \in \mathbb{R}$, cf. Lemma 2.6.

3.4 Characterization of the image of exp. In this subsection, we finally characterize the image of the exponential map modulo smoothing operators.

We use the fact that the algebra $L^{0}(X ; \Lambda)$ admits a holomorphic functional calculus, cf. [3, 22]. Therefore, for $B \in L^{0}(X ; \Lambda), \exp B$ is equally defined by the holomorphic functional calculus, i.e.,

$$
\exp B=\frac{1}{2 \pi i} \oint_{\mathcal{C}} e^{z}(z-B)^{-1} d z
$$

where $\mathcal{C}$ is a curve surrounding the spectrum of $B$ counter-clockwise. Moreover, if $\mathcal{J}$ is a closed ideal in $L^{0}(X ; \Lambda)$, then $\mathbb{C}+\mathcal{J}$ is a closed and inversely closed subalgebra of $L^{0}(X)$. Thus the holomorphic functional calculus leaves this subalgebra invariant. In particular, if $H \in \mathcal{J}$, then $\exp H=1+K$ for some $K \in \mathcal{J}$.

In case $l=0$ and $\mathcal{J}=L^{\mu}(X)$, where $\mu \in(-1-\mathbb{N}) \cup\{-\infty\}$, the reverse direction holds also true.

Lemma 3.24. Let $K \in L^{\mu}(X)$, where $\mu \in(-1-\mathbb{N}) \cup\{-\infty\}$. Suppose that $1+K \in \operatorname{Inv} L^{0}(X)$. Then there exists a $H \in L^{\mu}(X)$ such that

$$
\exp H=1+K
$$


Proof. The spectrum $\Sigma_{\mathcal{L}\left(H^{0}(X)\right)}(1+K)$ without the point 1 consists of finitely many eigenvalues of finite multiplicity possibly accumulating at 1 . Choose a ray $R_{\vartheta}=\left\{r e^{i \vartheta} ; 0 \leq r<\infty\right\}$, where $\vartheta \in(0,2 \pi)$ that does not meet $\Sigma_{\mathcal{L}\left(H^{0}(X)\right)}(1+K)$. Choose the branch of $\mathbb{C} \backslash R_{\vartheta} \ni z \mapsto \log z$ (e.g., such that $\log z \in \mathbb{R}$ for $z>0$ ) and define $\log (1+K)$ by the holomorphic functional calculus, i.e.,

$$
\log (1+K)=\frac{1}{2 \pi i} \oint_{\mathcal{C}} \log z(z-(1+K))^{-1} d z
$$

where $\mathcal{C}$ is a curve surrounding $\Sigma_{\mathcal{L}\left(H^{0}(X)\right)}(1+K)$ counter-clockwise.

By the remark above, we have

$$
\log (1+K)=c+H
$$

for a constant $c \in \mathbb{C}$ and $H \in L^{\mu}(X)$. By comparison of the principal symbols $\sigma^{0}(1+K)=1$ and $\sigma^{0}(c+H)=e^{c}$, we get that $c=2 \pi i k$ for some $k \in \mathbb{Z}$. Therefore,

$$
\exp H=\exp (c+H)=1+K
$$

as required.

Proposition 3.25. Let $A \in L^{\mu}(X)$. Then $A \in \operatorname{Exp} L^{\mu}(X)$ if and only if $A \in$ Inv $L^{\mu}(X)$ and $\Psi(A)=0$.

Proof. The conditions stated in the proposition are obviously necessary. Let us prove that these conditions are also sufficient.

Let $A \in \operatorname{Inv} L^{\mu}(X)$ and $\Psi(A)=0$. By Remark $3.19(\mathrm{a})$, we find a function $b \in S^{(\mu \log )}\left(T^{*} X \backslash 0\right)$ such that $\exp b=\sigma^{\mu}(A)$. Employing the short exact sequence (3.4), there is an operator $B_{1} \in L^{\mu \log }(X)$ such that $\sigma^{\mu \log }\left(B_{1}\right)=b$. Then $A \exp \left(-B_{1}\right)$ is invertible and has the form $1+K$ with $K \in L^{-1}(X)$. By Lemma 3.24, there is a $H \in L^{-1}(X)$ such that $\exp H=1+K$, i.e., $A=$ $\exp \left(B_{1}\right) \exp H \in \operatorname{Exp} L^{\mu}(X)$.

Remark 3.26. The foregoing proof also shows that each operator in $\operatorname{Exp} L^{\mu}(X)$ is the product of at most two exponentials, one of them being of the special form $\exp H=1+K$ (where even $H, K \in L^{-\infty}(X)$ can be arranged).

For $l \geq 1$, Lemma 3.24 is not valid (this is already seen in the scalar case $\operatorname{dim} X=$ $0)$. The formal reason for that is that an operator $K=K(\lambda) \in L^{\mu}(X ; \Lambda)$, where $\mu \in(-1-\mathbb{N}) \cup\{-\infty\}$, need not be compact as an operator in $H^{0}(X ; \Lambda)$. (The embedding $H^{s}(X ; \Lambda) \subset H^{s^{\prime}}(X ; \Lambda)$ for $s>s^{\prime}$ is not compact.) Nevertheless, we have the following substitute for Lemma 3.24:

Lemma 3.27. Let $K=K(\lambda) \in L^{\mu}(X ; \Lambda)$, where $\mu \in-1-\mathbb{N}$. Then there exists an operator $H=H(\lambda) \in L^{\mu}(X ; \Lambda)$ such that

$$
\exp H=1+K \quad \bmod L^{-\infty}(X ; \Lambda) .
$$


Proof. Recall from Lemma 2.2 that

$$
\Sigma_{\mathcal{L}\left(H^{0}(X ; \Lambda)\right)}(1+K)=\bigcup_{\lambda \in \Lambda} \Sigma_{\mathcal{L}\left(H^{0}(X)\right)}(1+K(\lambda))
$$

Moreover, $\|K(\lambda)\|_{\mathcal{L}\left(H^{0}(\lambda)\right)} \rightarrow 0$ as $|\lambda| \rightarrow \infty$. Therefore, there is a $\delta>0$ such that $\|K(\lambda)\|_{\mathcal{L}\left(H^{\circ}(X)\right)} \leq 1 / 4$ for all $|\lambda| \geq \delta$. In particular, for $|\lambda| \geq \delta, 1+K(\lambda)$ is invertible and

$$
\Sigma_{\mathcal{L}\left(H^{0}(X)\right)}(1+K(\lambda)) \subset B_{1 / 4}(1),
$$

where $B_{1 / 4}(1)=\{z \in \mathbb{C} ;|z-1| \leq 1 / 4\}$. For $|\lambda| \leq \delta, \Sigma_{\mathcal{L}\left(H^{0}(X)\right)}(1+K(\lambda)) \backslash$ $B_{1 / 4}(1)$ consists of finitely many eigenvalues of finite multiplicity. By perturbation theory (cf. [9]), these eigenvalues constitute continuously parametrized surfaces $\{\lambda \in \Lambda ;|\lambda| \leq \delta\} \rightarrow \mathbb{C}, \lambda \mapsto \gamma_{j}(\lambda)$, for $j=1, \ldots, \kappa$ and some $\kappa \in \mathbb{N}$ (it is possible that $\gamma_{j}(\lambda) \in B_{1 / 4}(1)$ for some $\lambda$ ). Thus

$$
\Sigma_{\mathcal{L}\left(H^{0}(X ; \Lambda)\right)}(1+K) \subset \bigcup_{j=1}^{\kappa}\left\{\gamma_{j}(\lambda) ;|\lambda| \leq \delta\right\} \cup B_{1 / 4}(1) .
$$

Thus even if $1+K$ is invertible, i.e., $\gamma_{j}(\lambda) \neq 0$ for all $|\lambda| \leq \delta$ and all $j$, it is possible that 0 does not belong to the unbounded component of $\mathbb{C} \backslash \Sigma_{\mathcal{L}\left(H^{0}(X ; \Lambda)\right)}(1+K)$. In that case the reasoning in the proof of Lemma 3.24 does not apply.

To prevent this situation, we remove part of the sets $\left\{\gamma_{j}(\lambda) ;|\lambda| \leq \delta\right\}, 1 \leq j \leq$ $\kappa$, from the spectrum of $1+K$ by adding an operator belonging to $L^{-\infty}(X ; \Lambda)$. This is achieved as follows: For $\lambda_{0} \in \Lambda, \varrho<1$, let $U\left(\lambda_{0}, \varrho\right)$ be, if it exists, an open neighbourhood of $\lambda_{0}$ in $\Lambda$ such that the finite part of $\Sigma_{\mathcal{L}\left(H^{0}(X)\right)}(1+K(\lambda))$ contained in $\{z \in \mathbb{C} ; \Re z \leq \varrho\}$ is non-empty and its algebraic multiplicity is constant for $\lambda \in U\left(\lambda_{0}, \varrho\right)$. Further let $\Lambda_{0}$ be the set of all $\lambda_{0} \in \Lambda$ for which there is a $U\left(\lambda_{0}, \varrho\right)$ for some $\varrho \leq 1 / 4$. We can suppose that $\Lambda_{0}$ is nonempty, otherwise we are already finished. $\Lambda_{0}$ has compact closure in $\Lambda$. Hence there are finitely many $\lambda_{1}, \ldots, \lambda_{h} \in \Lambda$ and corresponding $\varrho_{1}, \ldots, \varrho_{h} \leq 1 / 2$ such that the open sets $U_{j}=U\left(\lambda_{j}, \varrho_{j}\right), 1 \leq j \leq h$, cover $\bar{\Lambda}_{0}$, i.e., $\bar{\Lambda}_{0} \subset \bigcup_{j=1}^{h} U\left(\lambda_{j}, \varrho_{j}\right)$. Let $\left\{\varphi_{j}\right\}_{1 \leq j \leq h}$ be a smooth partition of unity subordinated to this covering. For $\lambda \in U_{j}$, let $\Pi_{j}(\lambda)$ be the part of the operator $1+K(\lambda)$ corresponding to the isolated part of $\Sigma_{\mathcal{L}\left(H^{0}(X)\right)}(1+K(\lambda))$ contained in $\left\{z \in \mathbb{C} ; \Re z \leq \varrho_{j}\right\}$. Then $\Pi_{j}(\lambda), \lambda \in U_{j}$, forms a smooth family of finite-rank operators of constant rank and with range in $C^{\infty}(X)$. Especially, $\Pi_{j} \in C^{\infty}\left(U_{j}, L^{-\infty}(X)\right)$. We set $\Pi(\lambda)=\sum_{j=1}^{h} \varphi_{j}(\lambda) \Pi_{j}(\Lambda), \lambda \in \Lambda$. Then $\Pi \in L^{-\infty}(X ; \Lambda)$ and $\|\Pi(\lambda)\|_{\mathcal{L}\left(H^{0}(X)\right)} \leq 1 / 2$ for all $\lambda \in \Lambda \backslash \Lambda_{0}$. In particular, 0 belongs to the unbounded component of $\mathbb{C} \backslash \Sigma_{\mathcal{L}\left(H^{0}(X ; \Lambda)\right)}(1+K-\Pi)$. Hence there is a logarithm $H \in L^{\mu}(X ; \Lambda)$ for the operator $1+K-\Pi \in 1+L^{\mu}(X ; \Lambda)$.

Henceforth, in view of Lemma 3.27, we shall work on the level of complete symbols, i.e., in the factor spaces $L^{\mu \log }(X ; \Lambda) / L^{-\infty}(X ; \Lambda)$ and $L^{\mu}(X ; \Lambda) / L^{-\infty}(X ; \Lambda)$, respectively, see Section 2.1.3 and Remark 3.5 (b). Notice that the exponential (1.2) 
map descents to a map

$$
\text { exp: } L^{\mu \log }(X ; \Lambda) / L^{-\infty}(X ; \Lambda) \rightarrow L^{\mu}(X ; \Lambda) / L^{-\infty}(X ; \Lambda) .
$$

Notice further that

$$
\operatorname{Ell} L^{\mu}(X ; \Lambda) / L^{-\infty}(X ; \Lambda)=\operatorname{Inv} L^{\mu}(X ; \Lambda) / L^{-\infty}(X ; \Lambda),
$$

see Lemma 2.3.

Compared to the situation of the map (1.2), see also Remark 3.17, we have:

Lemma 3.28. The image of the map (3.16) in $\bigcup_{\mu \in \mathbb{C}} L^{\mu}(X ; \Lambda) / L^{-\infty}(X ; \Lambda)$, i.e., when $\mu$ runs through $\mathbb{C}$, is already a group.

Proof. The single terms in the Campbell-Hausdorff formula are of orders decreasing to $-\infty$, cp. Remark 3.5 (c). Arguing modulo $L^{-\infty}(X ; \Lambda)$, we can asymptotically sum up all these terms. (Especially, no convergence problems arise.)

Analogously to Proposition 3.25 we have:

Proposition 3.29. Let $A \in \operatorname{Ell} L^{\mu}(X ; \Lambda)$. Then the class of $A$ in the factor space $L^{\mu}(X ; \Lambda) / L^{-\infty}(X ; \Lambda)$ belongs to the image of the map (3.16) if and only if $\Psi(A)=$ 0 .

Proof. Apply the same reasoning as in the proof of Proposition 3.25, now modulo $L^{-\infty}(X ; \Lambda)$.

\section{The KERNEL CUT-OFF TECHNiQUe}

The kernel cut-off technique has been introduced by Schulze to produce, starting from a given symbol, symbols which are holomorphic in one covariable. See [16, 17].

Let $\rho \in C_{0}^{\infty}\left(\mathbb{R}_{+}\right), \rho(1)=1$. Then the kernel cut-off operator $H(\rho)$ is defined by

$$
H(\rho) A(z)=M_{t \rightarrow z}\left\{\rho(t) M_{z^{\prime} \rightarrow t}^{-1}\left\{A\left(z^{\prime}\right)\right\}\right\},
$$

where $A(z)$ is an operator-valued function on the line $\Gamma_{\beta}=\{z \in \mathbb{C} ; \Re z=\beta\}$, $\beta \in \mathbb{R}$, and $M u(z)=\tilde{u}(z)=\int_{0}^{\infty} t^{z-1} u(t) d t$ is the Mellin transform with inverse $M^{-1} v(t)=(2 \pi i)^{-1} \int_{\Gamma_{\beta}} t^{-z} v(z) d z$.

It is known that $H(\rho)$ extends by continuity to an operator

$$
H(\rho): L^{\mu}\left(X ; \Gamma_{\beta}\right) \rightarrow \mathcal{M}_{\mathcal{O}}^{\mu}(X) .
$$

Furthermore,

$$
A-\left.H(\rho) A\right|_{\Gamma_{\beta}} \in L^{-\infty}\left(X ; \Gamma_{\beta}\right)
$$

for any $A \in L^{\mu}\left(X ; \Gamma_{\beta}\right)$. (4.1), (4.2) remain valid for $\mathcal{M}_{\mathcal{O}}^{\mu}(X), L^{\mu}\left(X ; \Gamma_{\beta}\right)$ replaced with $\mathcal{N}_{\mathcal{O}}^{\mu}(X), L_{1,0}^{\mu}\left(X ; \Gamma_{\beta}\right)$, cf. the notation introduced at the end of Section 2.2.

Henceforth, the function $\rho \in C_{0}^{\infty}\left(\mathbb{R}_{+}\right), \rho(1)=1$, shall be fixed. 
Proposition 4.1. Let $B \in L^{\mu \log }\left(X ; \Gamma_{\beta}\right), \beta \in \mathbb{R}$. Then

$$
H(\rho) B \in \mathcal{M}_{\mathcal{O}}^{\mu \log }(X)
$$

and

$$
B-\left.H(\rho) B\right|_{\Gamma_{\beta}} \in L^{-\infty}\left(X ; \Gamma_{\beta}\right) .
$$

Proof. Since $B \in L_{1,0}^{\varepsilon}\left(X ; \Gamma_{\beta}\right)$ for any $\varepsilon>0, H(\rho) B$ is well-defined and belongs to $\bigcap_{\varepsilon>0} \mathcal{N}_{\mathcal{O}}^{\varepsilon}(X)$. Furthermore, (4.4) holds.

To prove (4.3), we first note that $\phi(H(\rho) B) \psi \in \mathcal{M}_{\mathcal{O}}^{-\infty}(X)$ for all $\phi, \psi \in$ $C^{\infty}(X)$ with $\operatorname{supp} \phi \cap \operatorname{supp} \psi=\emptyset$, since $H(\rho) B \in \mathcal{N}_{\mathcal{O}}^{\varepsilon}(X)$ for $\varepsilon>0$. Hence it remains to show that $\kappa_{*} H(\rho) B=H(\rho)\left(\kappa_{*} B\right) \in \mathcal{M}_{\mathcal{O}}^{\mu \log }\left(\tilde{\Omega}_{\kappa}\right)$ for any coordinate chart $\kappa: \Omega_{\kappa} \rightarrow \tilde{\Omega}_{\kappa}$, where $\Omega_{\kappa}$ can be assumed to be simply connected, cf. Remark 3.3.

We can directly work on the level of symbols $b_{\kappa}(x, \xi, z) \in S^{\mu \log }\left(\tilde{\Omega}_{\kappa} \times \mathbb{R}^{n} \times \Gamma_{\beta}\right)$ instead of working with $\kappa_{*} B$ itself, i.e., we are allowed to assume that $\left(\kappa_{*} B\right)(z)=$ op $\left(b_{\kappa}(x, \xi, z)\right), z \in \Gamma_{\beta}$. By Lemma 3.1, we have

$$
\nabla_{\xi, z} b_{\kappa}(x, \xi, z) \in S^{-1}\left(\tilde{\Omega}_{\kappa} \times \mathbb{R}^{n} \times \Gamma_{\beta} ; \mathbb{C}^{n+1}\right), \quad \sigma^{0}\left(\xi \cdot \nabla_{\xi} b_{\kappa}+z \cdot \nabla_{z} b_{\kappa}\right)=\mu .
$$

Moreover,

$$
H(\rho) B_{\kappa}(z)=\text { op }\left(H(\rho) b_{\kappa}(x, \xi, z)\right),
$$

where $H(\rho)$ acts on $b_{\kappa}(x, \xi, z)$ in the covariable $z \in \Gamma_{\beta}$.

Let $b_{1 \kappa}(x, \xi, z)=H(\rho) b_{\kappa}(x, \xi, z), z \in \mathbb{C}$. Then

$$
b_{\kappa}(x, \xi, z)-\left.b_{1 \kappa}(x, \xi, z)\right|_{\Gamma_{\beta}} \in S^{-\infty}\left(\tilde{\Omega}_{\kappa} \times \mathbb{R}^{n} \times \Gamma_{\beta}\right) .
$$

Furthermore,

since

$$
\frac{\partial}{\partial z} H(\rho) b_{\kappa}(x, \xi, z)=H(\rho) \frac{\partial b_{\kappa}}{\partial z}(x, \xi, z)
$$

$$
H(\rho) b_{\kappa}(x, \xi, z)=\frac{1}{2 \pi i} \int_{\Gamma_{\beta}} \tilde{\rho}\left(z-z^{\prime}\right) b_{\kappa}\left(x, \xi, z^{\prime}\right) d z^{\prime}
$$

is a convolution and $\tilde{\rho}(z) \in \mathcal{S}\left(\Gamma_{\beta}\right),(2 \pi i)^{-1} \int_{\Gamma_{\beta}} \tilde{\rho}(z) d z=1$, i.e.,

$$
\frac{\partial b_{1 \kappa}}{\partial z}(x, \xi, z) \in S^{-1}\left(\tilde{\Omega}_{\kappa} \times \mathbb{R}^{n} \times \mathbb{C}_{\mathrm{hol}}\right)
$$

by the analogue of (4.1). Likewise, $\nabla_{\xi} b_{1 \kappa}(x, \xi, z)=H(\rho) \nabla_{\xi} b_{\kappa}(x, \xi, z) \in S^{-1}\left(\mathbb{R}^{n} \times\right.$ $\left.\mathbb{R}^{n} \times \mathbb{C}_{\text {hol }}\right)$.

The principal symbol of $\left.\nabla_{\xi, z} b_{1 \kappa}(x, \xi, z)\right|_{\Gamma_{\beta^{\prime}}}$ is independent of $\beta^{\prime}$, cf. Lemma 2.7. Therefore, for all $\beta^{\prime} \in \mathbb{R}$, we obtain in view of (4.5), (4.6)

$$
\sigma^{0}\left(\left.\left(\xi \cdot \nabla_{\xi} b_{1 \kappa}+z \cdot \nabla_{z} b_{1 \kappa}\right)\right|_{\Gamma_{\beta^{\prime}}}\right)=\mu \text {. }
$$

By Lemma 3.1, this shows that we indeed have $b_{1 \kappa}(x, \xi, z)=H(\rho) b_{\kappa}(x, \xi, z) \in$ $S^{\mu \log }\left(\tilde{\Omega}_{\kappa} \times \mathbb{R}^{n} \times \mathbb{C}_{\text {hol }}\right)$, i.e., $H(\rho) B_{\kappa} \in \mathcal{M}_{\mathcal{O}}^{\mu \log }\left(\tilde{\Omega}_{\kappa}\right)$. 
Corollary 4.2. Let $B \in L^{\mu \log }\left(X ; \Gamma_{\beta}\right), \beta \in \mathbb{R}$. Then we have

$$
\exp (H(\rho) B) \in \mathcal{M}_{\mathcal{O}}^{\mu}(X)
$$

and

$$
\exp B-\left.\exp (H(\rho) B)\right|_{\Gamma_{\beta}} \in L^{-\infty}\left(X ; \Gamma_{\beta}\right)
$$

Proof. This follows from Proposition 4.1, the fact that exp: $\mathcal{M}_{\mathcal{O}}^{\mu \log }(X) \rightarrow \mathcal{M}_{\mathcal{O}}^{\mu}(X)$, and

$$
\exp \left(\left.H(\rho) B\right|_{\Gamma_{\beta}}\right)=\exp (B+H)=\exp B+K
$$

for suitable $K, H \in L^{-\infty}\left(X ; \Gamma_{\beta}\right)$.

\section{ProOf OF THE MAIN THEOREM}

Recall from Section 2.3 that it suffices to prove Theorem 1.1 for holomorphic Mellin symbols. We then start the proof with the following simple observation:

If the conclusion holds for $G \in \operatorname{Ell} \mathcal{M}_{\mathcal{O}}^{\mu}(X), G^{\prime} \in \operatorname{Ell} \mathcal{M}_{\mathcal{O}}^{\mu^{\prime}}(X)$, then it also holds for $G G^{\prime} \in$ Ell $\mathcal{M}_{\mathcal{O}}^{\mu+\mu^{\prime}}(X)$. Indeed, if

$$
G=F(1+H), \quad G^{\prime}=F^{\prime}\left(1+H^{\prime}\right),
$$

where $F \in \operatorname{Inv} \mathcal{M}_{\mathcal{O}}^{\mu}(X), F^{\prime} \in \operatorname{Inv} \mathcal{M}_{\mathcal{O}}^{\mu^{\prime}}(X)$, and $H, H^{\prime} \in \mathcal{M}_{\mathcal{O}}^{-\infty}(X)$, then

$$
G G^{\prime}=F F^{\prime}\left(1+H_{1}\right)\left(1+H^{\prime}\right)=F F^{\prime}\left(1+H_{1}+H^{\prime}+H_{1} H^{\prime}\right)
$$

where $F F^{\prime} \in \operatorname{Inv} \mathcal{M}_{\mathcal{O}}^{\mu+\mu^{\prime}}(X), H_{1}=F^{\prime-1} H F^{\prime} \in \mathcal{M}_{\mathcal{O}}^{-\infty}(X)$, and $H_{1}+H^{\prime}+H_{1} H^{\prime} \in$ $\mathcal{M}_{\mathcal{O}}^{-\infty}(X)$.

This observation allows us to divide the proof into three steps.

5.1 Beginning of the proof. First we assume that $\Psi(G)=0$. Let $\beta \in \mathbb{R}$ be arbitrary. By Proposition 3.29, there is a $K \in L^{-\infty}\left(X ; \Gamma_{\beta}\right)$ such that $\left.G\right|_{\Gamma_{\beta}}+$ $K \in \operatorname{Exp} L^{\mu}\left(X ; \Gamma_{\beta}\right)$ is in the image of the exponential map exp: $L^{\mu \log }\left(X ; \Gamma_{\beta}\right) \rightarrow$ $L^{\mu}\left(X ; \Gamma_{\beta}\right)$. Choosing an arbitrary logarithm, we may apply the result of Proposition 4.1 and its Corollary 4.2 to obtain

$$
G-\exp H(\rho)\left[\log \left(\left.G\right|_{\Gamma_{\beta}}+K\right)\right] \in \mathcal{M}_{\mathcal{O}}^{-\infty}(X)
$$

for any $\rho \in C_{0}^{\infty}\left(\mathbb{R}_{+}\right), \rho(1)=1$. Obviously, $\exp H(\rho)\left[\log \left(\left.G\right|_{\Gamma_{\beta}}+K\right)\right] \in \operatorname{Inv} \mathcal{M}_{\mathcal{O}}^{\mu}(X)$ which completes the proof in this case. 
5.2 Continuation of the proof. Now we turn to the case of a general $\Psi(G)$. The quotient space

$$
\operatorname{Ell} \mathcal{M}_{\mathcal{O}}^{\mu}(X) /\left\{G \in \operatorname{Ell} \mathcal{M}_{\mathcal{O}}^{\mu}(X) ; \Psi(G)=0\right\} \cong H^{1}\left(\left(T^{*} X \times \mathbb{R}\right) \backslash 0 ; \mathbb{Z}\right)
$$

is described in the next lemma.

Lemma 5.1. We have

$$
H^{1}\left(\left(T^{*} X \times \mathbb{R}\right) \backslash 0 ; \mathbb{Z}\right)= \begin{cases}0 & \text { if } \operatorname{dim} X=0 \\ H^{1}(X ; \mathbb{Z}) \oplus \mathbb{Z} & \text { if } \operatorname{dim} X=1, \\ H^{1}(X ; \mathbb{Z}) & \text { if } \operatorname{dim} X \geq 2\end{cases}
$$

Proof. Using the homotopy sequence for the fibration $S^{*}(X \times \mathbb{R}) \stackrel{S^{n+1}}{\longrightarrow} X \times \mathbb{R}$, where $n=\operatorname{dim} X$ (and $S^{*}(X \times \mathbb{R})$ is the sphere bundle of $\left.\left(T^{*} X \times \mathbb{R}\right) \backslash 0 \rightarrow X\right)$, the circumstance that $S^{*}(X \times \mathbb{R})$ and $\left(T^{*} X \times \mathbb{R}\right) \backslash 0$ are homotopy equivalent, and the fact that

$$
\operatorname{Hom}\left(\pi_{1}(Y), \mathbb{Z}\right)=\operatorname{Hom}\left(H_{1}(Y), \mathbb{Z}\right)=H^{1}(Y, \mathbb{Z}),
$$

where $Y=\left(T^{*} X \times \mathbb{R}\right) \backslash 0$, we arrive at (5.2).

From Section 5.1 it follows that in case $\operatorname{dim} X=0$ we are already done. Furthermore, every class in $H^{1}(X ; \mathbb{Z})$ in the direct decomposition of $H^{1}\left(\left(T^{*} X \times \mathbb{R}\right) \backslash 0 ; \mathbb{Z}\right)$ according to (5.2) is, in view of Lemma 3.18, realized by some multiplication operator $M_{\psi}$, where $\psi \in C^{\infty}(X)$ and $\psi(x) \neq 0$ for all $x \in X$. Since such an operator gives rise to a Mellin symbols in Inv $\mathcal{M}_{\mathcal{O}}^{0}(X)$, every other Mellin symbol $G$ with $\Psi(G) \in H^{1}(X ; \mathbb{Z})$ can be reduced with the help of a multiplication operator to the case $\Psi(G)=0$ that has been settled before. That means that we are also done in case $\operatorname{dim} X \geq 2$, while for $\operatorname{dim} X=1$ it remains to factorize an operator whose $\Psi$-invariant realizes the generator 1 in the second summand $\mathbb{Z}$ in the direct decomposition $(5.2)$ of $H^{1}\left(\left(T^{*} X \times \mathbb{R}\right) \backslash 0 ; \mathbb{Z}\right)$.

5.3 The remaining case for $\operatorname{dim} \mathbf{X}=1$. For $\operatorname{dim} X=1, X$ is diffeomorphic to the circle $S^{1}=\{\zeta \in \mathbb{C} ;|\zeta|=1\}$. We introduce $\phi$ with $\zeta=e^{i \phi}$ as local coordinate on $S^{1}, \phi \in[0,2 \pi]$.

Let

$$
(u, v)=\frac{1}{2 \pi} \int_{0}^{2 \pi} u(\phi) \overline{v(\phi)} d \phi
$$

be the scalar-product in $H^{0}\left(S^{1}\right)$. Then $\left\{e^{i k \phi} ; k \in \mathbb{Z}\right\}$ is an orthonormal basis in $H^{0}\left(S^{1}\right)$. Furthermore, for $s \in \mathbb{R}$,

$$
\|u\|_{s}=\left\{\sum_{k \in \mathbb{Z}}\left(1+k^{2}\right)^{s}\left|u_{k}\right|^{2}\right\}^{1 / 2},
$$


where $u=\sum_{k \in \mathbb{Z}} u_{k} e^{i k \phi}$, i.e., $u_{k}=\left(u, e^{i k \phi}\right)$, defines the norm in $H^{s}\left(S^{1}\right)$. For $k \in \mathbb{Z}$, let $\Pi_{k}$ be the orthogonal projection in $H^{0}\left(S^{1}\right)$ onto the subspace spanned by $e^{i k \phi}$. Then

$$
\left\|\Pi_{k}\right\|_{H^{s}\left(S^{1}\right) \rightarrow H^{s^{\prime}\left(S^{1}\right)}}=\langle k\rangle^{s^{\prime}-s}
$$

for all $s, s^{\prime} \in \mathbb{R}$.

Now it is seen that

$$
z+D_{\phi}=\sum_{k \in \mathbb{Z}}(z+k) \Pi_{k} \in \mathcal{M}_{\mathcal{O}}^{1}\left(S^{1}\right)
$$

is a differential operator with $\Psi\left(z+D_{\phi}\right)$ being equal to the generator 1 in the second summand $\mathbb{Z}$ in the direct decomposition of $H^{1}\left(\left(T^{*} X \times \mathbb{R}\right) \backslash 0 ; \mathbb{Z}\right)$. Hence it suffices to factorize this operator.

Lemma 5.2. Let $k \in \mathbb{Z}, k \neq 0, h \in \mathbb{N}$, and $\alpha>0$. Then there is a function $g(z) \in \mathcal{M}_{\mathcal{O}}^{-\infty}$ such that $z+k+g(z) \in \operatorname{Inv} \mathcal{M}_{\mathcal{O}}^{1}$ and, in addition,

$$
\langle z\rangle^{j}\left|\partial_{z}^{j} g(z)\right| \leq \alpha \text { if }|\Re z| \leq|k|-1, j \leq h
$$

Proof. The $\operatorname{logarithm} \log (z+k)$ can be defined for $|\Re z| \leq|k|-1$, since $z+k$ has no zeros in that region, and then, in particular, $\left.\log (z+k)\right|_{\Gamma_{\beta}} \in S^{\log }\left(\Gamma_{\beta}\right)$ for $\beta \in \mathbb{R},|\beta| \leq|k|-1$. Therefore, choosing $\rho \in C_{0}^{\infty}\left(\mathbb{R}_{+}\right), \rho(1)=1$, and defining $\rho_{\epsilon} \in C_{0}^{\infty}\left(\mathbb{R}_{+}\right), \rho_{\epsilon}(1)=1$, by $\tilde{\rho}_{\epsilon}(z)=\epsilon^{-1} \tilde{\rho}\left(\epsilon^{-1} z\right)$ for $\epsilon>0$, we have

$$
\left.\left.\left(H\left(\rho_{\epsilon}\right) \log (z+k)\right)\right|_{\Gamma_{\beta}} \rightarrow \log (z+k)\right|_{\Gamma_{\beta}} \text { in } S^{\log }\left(\Gamma_{\beta}\right) \text { as } \epsilon \rightarrow 0^{+},
$$

i.e.,

$$
\left.\left.\left(\exp H\left(\rho_{\epsilon}\right) \log (z+k)\right)\right|_{\Gamma_{\beta}} \rightarrow(z+k)\right|_{\Gamma_{\beta}} \text { in } S^{1}\left(\Gamma_{\beta}\right) \text { as } \epsilon \rightarrow 0^{+}
$$

uniformly for $\beta \in \mathbb{R},|\beta| \leq|k|-1$. Moreover, $z+k-\exp H\left(\rho_{\epsilon}\right) \log (z+k) \in \mathcal{M}_{\mathcal{O}}^{-\infty}$, i.e.,

$$
\left.(z+k)\right|_{\Gamma_{\beta}}-\left.\left(\exp H\left(\rho_{\epsilon}\right) \log (z+k)\right)\right|_{\Gamma_{\beta}} \rightarrow 0 \text { in } S^{-\infty}\left(\Gamma_{\beta}\right) \text { as } \epsilon \rightarrow 0^{+}
$$

uniformly for $\beta \in \mathbb{R},|\beta| \leq|k|-1$. If $\epsilon>0$ is sufficiently small, then the assertion follows.

Proposition 5.3. There exists a $f(z) \in \operatorname{Inv} \mathcal{M}_{\mathcal{O}}^{1}\left(S^{1}\right)$ such that

$$
z+D_{\phi}-f(z) \in \mathcal{M}_{\mathcal{O}}^{-\infty}\left(S^{1}\right)
$$


Proof. Choose a sequence $\left\{\alpha_{k}\right\}_{k \in \mathbb{Z}}$ of positive numbers that is rapidly decreasing. According to the foregoing lemma, choose functions $g_{k}(z) \in \mathcal{M}_{\mathcal{O}}^{-\infty}$ such that $z+k+g_{k}(z) \in \operatorname{Inv} \mathcal{M}_{\mathcal{O}}^{1}$ and

$$
\langle z\rangle^{j}\left|\partial_{z}^{j} g_{k}(z)\right| \leq \alpha_{k} \text { if }|\Re z| \leq|k|-1, j \leq|k|-1 \text {. }
$$

Then we set

$$
f(z)=\sum_{k \in \mathbb{Z}}\left(z+k+g_{k}(z)\right) \Pi_{k}
$$

It suffices to prove that

$$
g(z)=\sum_{k \in \mathbb{Z}} g_{k}(z) \Pi_{k} \in \mathcal{M}_{\mathcal{O}}^{-\infty}\left(S^{1}\right)
$$

since $f(z)$ is everywhere invertible and we then have $f(z) \in \operatorname{Inv} \mathcal{M}_{\mathcal{O}}^{1}\left(S^{1}\right)$.

For $h \in \mathbb{N},|\Re z| \leq h, j \leq h$, and $s, s^{\prime} \in \mathbb{R}$, we estimate

$$
\begin{aligned}
\langle z\rangle^{j}\left\|\partial_{z}^{j} g(z)\right\|_{H^{s} \rightarrow H^{s^{\prime}}} & \leq \sum_{|k| \leq h}\langle z\rangle^{j}\left|\partial_{z}^{j} g_{k}(z)\right|\langle k\rangle^{s^{\prime}-s}+\sum_{|k|>h}\langle z\rangle^{j}\left|\partial_{z}^{j} g_{k}(z)\right|\langle k\rangle^{s^{\prime}-s} \\
& \leq \sum_{|k| \leq h}\langle z\rangle^{j}\left|\partial_{z}^{j} g_{k}(z)\right|\langle k\rangle^{s^{\prime}-s}+\sum_{|k|>h}\langle k\rangle^{s^{\prime}-s} \alpha_{k}
\end{aligned}
$$

i.e.,

$$
\sup _{|\Re z| \leq h, j \leq h}\langle z\rangle^{j}\left\|\partial_{z}^{j} g(z)\right\|_{H^{s}\left(S^{1}\right) \rightarrow H^{s^{\prime}\left(S^{1}\right)}}<\infty
$$

showing that $g(z) \in \mathcal{M}_{\mathcal{O}}^{-\infty}\left(S^{1}\right)$.

The proof of Theorem 1.1 is complete.

\section{REFERENCES}

1. R. Beals, Characterization of pseudodifferential operators and applications, Duke Math. J. 44 (1977), 45-57.

2. H. O. Cordes, The Technique of Pseudodifferential Operators, London Math. Soc. Lecture Note Ser., vol. 202, Cambridge Univ. Press, Cambridge, 1995.

3. B. Gramsch, Relative Inversion in der Störungstheorie von Operatoren und $\Psi$-Algebren, Math. Ann. 269 (1984), 27-71.

4. B. Gramsch and W. Kaballo, Multiplicative decomposition of holomorphic Fredholm functions and $\Psi^{*}$-algebras, Math. Nachr. 204 (1999), 83-100.

5. G. Grubb and R. T. Seeley, Weekly parametric pseudodifferential operators and AtiyahPatodi-Singer boundary problems, Invent. Math. 121 (1995), 481-529.

6. M. Hausner and J. Schwartz, Lie Groups; Lie Algebras, Notes on Math. Appl., Gordon and Breach, New York, 1968.

7. L. Hörmander, The Analysis of Linear Differential Operators III, IV, Grundlehren Math. Wiss., vol. 274, 275, Springer, Berlin, 1985. 
8. D. Kapanadze, B.-W. Schulze, and I. Witt, Coordinate invariance of the cone algebra with asymptotics (this volume).

9. T. Kato, Perturbation Theory for Linear Operators, Grundlehren Math. Wiss., vol. 132, Springer, Berlin, 1976.

10. M. Kontsevich and S. Vishik, Geometry of determinants of elliptic operators, Functional Analysis on the Eve of the 21st Century, Volume I. In Honor of the Eightieth Birthday of I. M. Gelfand (S. Gindikin, J. Lepowsky, and R. L. Wilson, eds.), Progress in Math., vol. 131, Birkhäuser, Boston, 1995, pp. 173-197.

11. X. Liu, A cone pseudodifferential calculus on the half-line with respect to conormal asymptotics of a given type, Ph.D. Thesis, Department of Mathematics, Wuhan University, Wuhan, 2000.

12. X. Liu and I. Witt, Asymptotic expansions for bounded solutions to semilinear Fuchsian equations, Preprint 2001/01, University of Potsdam, Institute of Mathematics, January 2001.

13. _ Pseudodifferential calculi on the half-line respecting prescribed asymptotic types (in preparation).

14. A. Pazy, Semigroups of Linear Operators and Applications to Partial Differential Equations, Appl. Math. Sci., vol. 44, Springer, New York, 1983.

15. B.-W. Schulze, Mellin expansions of pseudo-differential operators and conormal asymptotics of solutions, Pseudo-differential Operators. Proceedings, Oberwolfach 1986 (H. O. Cordes, B. Gramsch, and H. Widom, eds.), Lecture Notes in Math., vol. 1256, Springer, Berlin, 1987, pp. 378-401.

16. _ Pseudo-Differential Operators on Manifolds with Singularities, Stud. Math. Appl., vol. 24, North Holland, Amsterdam, 1991.

17. _ Boundary Value Problems and Singular Pseudo-differential Operators, Wiley Ser. Pure Appl. Math., J. Wiley, Chichester, 1998.

18. _ Pseudo-differential calculus and applications to non-smooth configurations, Lecture Notes of TICMI 1 (2000), Tbilisi University Press, Tbilisi.

19. R. T. Seeley, Complex powers of an elliptic operator, Singular Integrals (A. Calderon, eds.), Proc. Sympos. Pure Math., vol. 10, Amer. Math. Soc., Providence, RI, 1967, pp. 288-307.

20. M. A. Shubin, Pseudodifferential Operators and Spectral Theory, Springer Ser. Soviet Math., Springer, Berlin, 1987.

21. N. Steenrod, The Topology of Fibre Bundles, Princeton Math. Ser., vol. 14, Princeton Univ. Press, Princeton, NJ, 1951.

22. L. Waelbroeck, Topological Vector Spaces and Algebras, Lecture Notes in Math., vol. 230, Springer, Berlin, 1971.

23. I. Witt, Explicit algebras with the Leibniz-Mellin translation product (submitted to Math. Nachr.).

University of Potsdam, Institute of Mathematics, PF 6015 53, D-14415 PotsDAM, GERMANY

E-mail address: ingo@math.uni-potsdam.de 